\title{
An Analysis of Unreliability and Asymmetry in Low-Power Wireless Links
}

\author{
MARCO ZÚÑIGA ZAMALLOA and BHASKAR KRISHNAMACHARI \\ University of Southern California
}

Experimental studies have demonstrated that the behavior of real links in low-power wireless net-
works (such as wireless sensor networks) deviates to a large extent from the ideal binary model
used in several simulation studies. In particular, there is a large transitional region in wireless
link quality that is characterized by significant levels of unreliability and asymmetry, significantly
impacting the performance of higher-layer protocols. We provide a comprehensive analysis of the
root causes of unreliability and asymmetry. In particular, we derive expressions for the distribu-
tion, expectation, and variance of the packet reception rate as a function of distance, as well as
for the location and extent of the transitional region. These expressions incorporate important en-
vironmental and radio parameters such as the path loss exponent and shadowing variance of the
channel, and the modulation, encoding, and hardware variance of the radios.

Categories and Subject Descriptors: C.2.1 [Computer-Communication Networks]: Network Architecture and Design—Wireless communication; I.6.1 [Simulation and Modeling]: Simulation Theory—Systems theory

General Terms: Measurement, Performance, Theory, Verification

Additional Key Words and Phrases: Wireless link, transitional region, hardware variance, communication theory, probability theory

ACM Reference Format:

Zúñiga Zamalloa, M. and Krishnamachari B. 2007. An analysis of unreliability and asymmetry in low-power wireless links. ACM Trans. Sensor Netw. 3, 2, Article 7 (June 2007), 34 pages. DOI = 10.1145/1240226.1240227 http://doi.acm.org/10.1145/1240226.1240227

\section{INTRODUCTION}

Wireless sensor network (WSN) protocols are often evaluated through simulations that make simplifying assumptions about the link layer, such as the ideal

This work has been supported in part by the National Science Foundation under Grants CANS0325875, CANS-0347621, CANS-0435505, and CC-0430061. An earlier version of this article appeared in the 2004 Proceedings of the IEEE SenCon (Santa Clara, CA).

Author's address: M. Zúñiga Zamalloa, B. Krishnamachari, Department of Electrical EngineeringSystems, University of Southern California, Los Angeles, CA 90089-0781; e-mail: \{marcozun, bkrishna\}@usc.edu.

Permission to make digital or hard copies part or all of this work for personal or classroom use is granted without fee provided that copies are not made or distributed for profit or direct commercial advantage and that copies show this notice on the first page or initial screen of a display along with the full citation. Copyrights for components of this work owned by others than ACM must be honored. Abstracting with credit is permitted. To copy otherwise, to republish, to post on servers, to redistribute to lists, or to use any component of this work in other works requires prior specific permission and/or a fee. Permissions may be requested from the Publications Dept., ACM, Inc., 2 Penn Plaza, Suite 701, New York, NY 10121-0701 USA, fax +1 (212) 869-0481, or permissions@acm.org. (C) 2007 ACM 1550-4859/2007/06-ART7 \$5.00 DOI 10.1145/1240226.1240227 http://doi.acm.org/ $10.1145 / 1240226.1240227$ 
binary model. In this model, packets are received only within the circular radio range of the transmitter. However, the real characteristics of low-power wireless links differ greatly from those of the ideal model; chiefly among these differences are the unreliable and asymmetric nature of real links. The significant differences between the ideal model and real behavior can lead to erroneous performance evaluation of upper-layer protocols (network layer and above).

Several studies [Ganesan et al. 2003; Zhao and Govindan 2003; Woo et al. 2003] have classified low-power wireless links in three distinct reception regions: connected, transitional, and disconnected. In the connected region, links are often of good quality, stable, and symmetric. By contrast, the transitional region is characterized by the presence of unreliable and asymmetric links; and the disconnected region presents no practical links for transmission. Unfortunately, the transitional region is often quite significant in size, and in dense deployments such as those envisioned for sensor networks, a large number of the links in the network (even greater than 50\% [Zhao and Govindan 2003]) can be unreliable.

Recent studies have shown that unreliable and asymmetric links can have a major impact on the performance of upper-layer protocols. In Ganesan et al. [2003], it is shown that the dynamics of even the simplest flooding mechanism can be significantly affected due to asymmetric and occasional long-distance links. In Kotz et al. [2003], it is argued that routing structures formed by taking into account unreliable links can be significantly different from structures based on the simple binary model. Similarly, the authors of Zhou et al. [2006] report that such unreliable links can have a negative impact on routing protocols, particularly geographic forwarding schemes.

Other works [Woo et al. 2003; De Couto et al. 2005] have proposed mechanisms to take advantage of nodes in the transitional region. For instance, the authors of De Couto et al. [2005] found that protocols using a traditional minimum hop-count metric perform poorly in terms of throughput, and that a new metric called ETX (expected number of transmissions), which uses nodes in the transitional region, has better performance.

The significant impact of real link characteristics on the performance of upper-layer protocols has created increased understanding for the need for realistic link-layer models for wireless sensor networks. In order to address this need, some recent works [Woo et al. 2003; Zhou et al. 2006; Cerpa et al. 2005] have proposed new link models based on empirical data. However, these models do not provide significant mathematical insight into how channel and radio dynamics affect link unreliability and asymmetry. Also, some of these works [Cerpa et al. 2005; Woo et al. 2003] are valid only for the specific channel and radio parameters used in the deployment.

In this study, we use analytical tools from communication theory, simulations, and experiments to present an in-depth analysis of unreliable and asymmetric links in low-power multihop wireless networks. The main contributions of this work are twofold. First, our work allows quantifying the impact of the wireless environment and radio characteristics on link reliability and asymmetry. Second, we propose a systematic way to generalize models for the link layer so as to enhance simulation accuracy. 
Table I. Organization

\begin{tabular}{lc}
\hline Topic & Section \\
\hline Related Work & 2 \\
Channel Dynamics & 3 \\
Link Model & 3.1 \\
Impact on Link Reliability & 3.2 \\
Expectation and Variance of PRR & 3.3 \\
Comparison with Available Models & 3.4 \\
Hardware Variance & 4 \\
Hardware Variance Model & 4.1 \\
Impact on Link Asymmetry & 4.2 \\
Impact on Link Reliability & 4.3 \\
Empirical Validation & 5 \\
\hline
\end{tabular}

We also derive expressions for both the packet reception rate as a function of distance, and for the size of the transitional region. These expressions incorporate several radio parameters such as modulation, encoding, output power, frame size, receiver noise floor, and hardware variance, as well as important channel parameters, namely, the path loss exponent and log-normal variance.

Table I presents the organization of the article. In Section 2, we present related work. Section 3 studies the impact of multipaths on link reliability. First, we present a model for the packet reception rate as a function of distance in Section 3.1. Based on this model, in Section 3.2 we study the impact of channel and radio parameters on link reliability by analyzing their effect on the extent of the transitional region. Then, in Section 3.3 we present approximate expressions for the expectation and variance of the packet reception rate as a function of distance. The section ends with a comparison of available link models with the one proposed in this work (Section 3.4).

We study the impact of hardware variance in Section 4. Hardware variance has already been identified as the cause of link asymmetry [Cerpa et al. 2005], and in addition, we also show that it can play a significant role on the extent of the transitional region. In Section 4.1, we present a model for hardware variance. Based on this model, the impact of hardware variance on link asymmetry and reliability is quantified in Sections 4.2 and 4.3, respectively. Finally, in Section 5 we present empirical measurements, based on a testbed of mica2 motes, which validate some analytical insights of our work. Our conclusions are presented in Section 6.

Before proceeding, we present the scope of our work. Our study is focused on static and neither low-dynamic environments and considers interference effects nor the nonisotropic property of radio coverage. However, it can be complemented with other research efforts to incorporate these dynamics. For instance, in Son et al. [2006] the authors focus on interference in wireless sensor networks, Cerpa et al. [2005] study some temporal properties and Zhou et al. [2006] provide an interesting model for the nonisotropic characteristic of radio coverage; the models presented in these works can be used to complement ours. Appendix A. A presents some guidelines on how to combine the nonisotropic RIM model [Zhou et al. 2006] with our work. 


\section{RELATED WORK}

The unrealistic nature of some common assumptions used in mobile ad hoc networks (MANET) research is presented in Kotz et al. [2003]. In real scenarios, packet losses lead to different connectivity graphs, and coverage ranges that are neither circular nor convex and often noncontiguous.

Several researchers have pointed out that simplistic assumptions may lead to erroneous performance evaluations of upper-layer protocols. In one of the earliest works [Ganesan et al. 2003], the authors present empirical results on the behavior of simple flooding in a dense sensor network. They found that the flooding tree exhibits high clustering behavior in contrast to the more uniformly distributed tree obtained with the ideal binary model.

In De Couto et al. [2005], the authors present measurements for DSDV and DSR over a 29 -node $802.11 \mathrm{~b}$ testbed and show that when real channel characteristics are not taken into account, the minimum hop-count metric has poor performance. By incorporating the effects of link loss ratios, asymmetry, and interference, they present the expected transmission count metric which finds high throughput paths. Along similar lines, Woo et al. [2003] study the effect of link connectivity on distance-vector-based routing in sensor networks. By evaluating link estimators, neighborhood table management, and reliable routing protocol techniques, they found that cost-based routing using a minimum expected transmission metric shows good performance.

Zhou et al. [2006] reported that radio irregularity has significant impact on routing protocols, but a relatively small impact on MAC protocols. They found that location-based routing protocols such as geographic routing perform worse in the presence of radio irregularity than on-demand protocols such as AODV and DSR.

Through empirical studies, the previous works bring to light the impact that unreliable and asymmetric links have on protocol performance at different layers. Nevertheless, while on-site deployment is arguably the best testing procedure for small-scale networks, it may be unfeasible for medium- and large-scale networks, for which simulators are usually the best option. In order to help overcome this problem some tools and models have been recently proposed to obtain more accurate link-layer models.

In Woo et al. [2003], the authors derive a packet loss model based on aggregate statistical measures such as the mean and standard deviation of the packet reception rate. The model assumes a Gaussian distribution of the packet reception rate for a given transmitter-receiver distance, which, it will be shown in Section 3, is not accurate.

Using the SCALE tool [Cerpa et al. 2003], Cerpa et al. [2005] identify other factors for link modeling. They capture features of the groups of links associated with a particular receiver and transmitter, as well as links associated with a group of radios in close proximity. Using several statistical techniques, they provide a model spectrum of increasing complexity and accuracy.

A more recent model, called the radio irregularity model (RIM), was proposed in Zhou et al. [2006]. Based on experimental data, RIM provides a radio model that takes into account both the nonisotropic properties of propagation 
media and the heterogeneous properties of devices to build a richer link model.

Temporal properties in dynamic environments have been studied as well. In Cerpa et al. [2005], the authors study short-term temporal issues such as autocorrelation of individual and reverse links, and long-term temporal properties such as the length of time needed to measure the channel and how often to obtain accurate link quality metrics. The authors also propose new routing algorithms to take advantage of the temporal properties of wireless links.

While the described models are important steps toward a realistic link quality model, they do not provide significant mathematical insight on how the channel and radio parameters affect link unreliability and asymmetry. Also, some of these models [Woo et al. 2003; Cerpa et al. 2005] do not provide a systematic way to generalize the models (i.e., extend their validity and accuracy) beyond the specific radio and environment conditions of the experiments from which the models are derived.

On the other hand, years of research in wireless communications, particularly cellular networks, provide a rich set of models and tools for analyzing the physical layer [Rappaport 2002].$^{1}$ Two of these tools are of significance to understand the transitional region, the log-normal path loss model (to model the wireless channel), and the bit-error performance of various modulation and encoding schemes with respect to the signal-to-noise ratio (to model the radio).

Hitherto, research has identified the link-layer modeling problem and its impact on upper-layer protocols. It also has identified some of its causes and proposed some realistic link quality models. However, what is missing is a clear analytical understanding of the root causes of link behavior. Our work presents an in-depth analysis of unreliable and asymmetric links and provides simple analytical models for the link layer.

\section{IMPACT OF CHANNEL DYNAMICS}

The extent of the transitional region is the result of placing specific devices, such as mica 2 motes, in a specific environment like the aisle of a building. If the characteristics of one of these elements is altered (radio or channel) then the extent of the transitional region is also altered. With the intent of analyzing how the channel and radio determine this extent, we first define models for both elements and subsequently study their interaction. Then we present approximated expressions for the expectation and variance of the packet reception rate with respect to distance. Finally, we compare our model with a previously proposed model.

From the network-layer perspective, a desired abstraction for link quality is the packet reception rate as a function of distance. This abstraction can be derived by composing the channel model, which provides the received signal strength (RSS) as a function of distance, with the radio-receiver model, which

${ }^{1}$ In cellular systems the transitional region is not of interest (except for modeling intercell interference), as cells are designed to fit only the connected region. 
Table II. Mathematical Notation

\begin{tabular}{lc}
\hline Description & Symbol \\
\hline Packet Reception Rate Parameters & \\
\hline - packet reception rate (PRR) & $\Psi$ \\
- a specific PRR value in the range of $\Psi$ & $\psi$ \\
- high PRR & $\psi_{h}$ \\
- low PRR & $\psi_{\ell}$ \\
\hline Signal to Noise Ratio Parameters & $\Upsilon$ \\
\hline - signal to noise ratio (SNR) & $\gamma$ \\
- a specific SNR value in the range of $\Upsilon$ & $\gamma_{h}$ \\
- SNR value corresponding to $\psi_{h}$ & $\gamma_{\ell}$ \\
- SNR value corresponding to $\psi_{\ell}$ & $\mu(d)$ \\
- mean of SNR (Gaussian) for distance $d$ & $\beta$ \\
- bit error rate as a function of SNR & $\mathcal{B}$ \\
- bit error rate as a function of SNR in dB & \\
\hline Channel Parameters & $\eta$ \\
- path loss exponent & $\sigma$ \\
- standard deviation & $P_{t}$ \\
- output power & $P_{r}$ \\
- received power & $P_{n}$ \\
- noise floor & $\mathcal{N}$ \\
- Gaussian random variable & \\
\hline Transitional Region Parameters & $\Gamma$ \\
- transitional region coefficient & $d_{b}$ \\
- beginning of transitional region & $d_{e}$ \\
- end of transitional region &
\end{tabular}

provides the packet reception rate (PRR) as a function of the signal-to-noise ratio (SNR).

In the remainder of the article, the SNR function is denoted by $\Upsilon$ and the PRR function by $\Psi$. Also, the lowercase greek letter $\gamma=\Upsilon($.) and $\psi=\Psi($.) represent values taken by $\Upsilon$ and $\Psi$, respectively, for specific points in their respective domains. Table II presents a summary of the notation used in this article.

\subsection{Channel and Radio Receiver Models}

-Channel: When an electromagnetic signal propagates, it may be diffracted, reflected, and scattered. These effects have two important consequences on the signal strength. First, the signal strength decays exponentially with respect to distance, and second, for a given distance $d$, the signal strength is random and log-normally distributed about the mean distance-dependent value.

Due to the unique characteristics of each environment, most radio propagation models use a combination of analytical and empirical methods. One of the most common radio propagation models is the log-normal path loss model [Rappaport 2002]. This model can be used for large and small coverage systems [Seidel and Rappaport]. Furthermore, empirical studies have shown that the log-normal model provides more accurate multipath channel models than the well-known Nakagami and Rayleigh models for indoor environments [Nikookar and Hashemi 1993]. 
According to this model, the received power $\left(P_{r}\right)$ in $\mathrm{dB}$ is given by

$$
P_{r}(d)=P_{t}-P L\left(d_{0}\right)-10 \eta \log _{10}\left(\frac{d}{d_{0}}\right)+\mathcal{N}(0, \sigma),
$$

where $P_{t}$ is the output power, $\eta$ is the path loss exponent that captures the rate at which the signal decays with respect to distance, $\mathcal{N}(0, \sigma)$ is a Gaussian random variable with mean 0 and variance $\sigma$ (standard deviation due to multipath effects), and $P L\left(d_{0}\right)$ is the power decay for the reference distance $d_{0}$.

Eq. (1) does not consider nonisotropic transmission, which is an important characteristic of low-power wireless links. In Appendix A we present some guidelines on how to incorporate these nonisotropic effects into our model by using the expressions derived in the RIM model [Zhou et al. 2006]. Appendix A also presents some information on how to include the path loss effect caused by obstacles.

Radio receiver. The receiver response is given by the packet reception rate as a function of the SNR. The packet reception rate can be derived from biterror rate expressions that are widely available in the wireless communication literature.

For a modulation $M$, the packet reception rate $(\Psi)$ is defined in terms of the bit-error rate $\left(\beta_{M}\right)$ as $^{2}$

$$
\Psi(\gamma)=\left(1-\beta_{M}(\gamma)\right)^{f},
$$

where $f$ is the number of bits transmitted, and step 3 in Table VII presents expressions of $\beta_{M}$ for some common narrowband modulation schemes.

$\beta_{M}$ is a function of the SNR. The SNR can be obtained from Eq. (1) and is given by

$$
\begin{aligned}
\Upsilon(d) & =P_{r}(d)-P_{n} \\
& =\mathcal{N}(\mu(d), \sigma),
\end{aligned}
$$

where $\mathcal{N}(\mu(d), \sigma)$ is a Gaussian random variable with mean $\mu(d)$, variance $\sigma^{2}$, and $P_{n}$ is the noise floor. ${ }^{3}$ Specifically, $\mu(d)$ can be derived by inserting Eq. (1) into Eq. (3), which leads to

$$
\mu(d)=P_{t}-P L\left(d_{0}\right)-10 \eta \log _{10}\left(\frac{d}{d_{0}}\right)-P_{n} .
$$

Given that the SNR in Eq. (3) is in $\mathrm{dB}$, let us redefine the packet reception rate in Eq. (2) as a function of the SNR in $\mathrm{dB}$. Denoting $\omega(x)=10^{x / 10}$ and the bit-error rate for $\mathrm{SNR}$ in $\mathrm{dB}$ as $\mathcal{B}_{M}\left(\gamma_{d B}\right)=\beta_{M}\left(\omega\left(\gamma_{d B}\right)\right)$, the packet reception rate $\Psi$ can be redefined as

$$
\Psi\left(\gamma_{d B}\right)=\left(1-\mathcal{B}_{M}\left(\gamma_{d B}\right)\right)^{f} .
$$

\footnotetext{
${ }^{2}$ For ease of explanation, the encoding is assumed to be NRZ. Table VII presents expressions for other encoding techniques.

${ }^{3}$ The focus of this article is on static interference-free environments. In these scenarios $P_{n}$ is given only by thermal noise and is constant, which in turn leads to constant packet reception rates in time, as shown in Son et al. [2004]. Nevertheless, in most scenarios $P_{n}$ changes with time, either because of interference or because of large changes in temperature. For these scenarios $P_{n}$ can be modeled as a random process.
} 
while the previous equation is general and valid for any modulation $M$, the figures in this section assume noncoherent FSK (NCFSK) modulation. The figures are for illustrative purposes and any modulation would serve that purpose. NCFSK was chosen because the empirical evaluation presented in Section 5 uses NCFSK radios (i.e., CC1000 equipped mica2 motes).

\subsection{Impact on Link Reliability (Extent of Transitional Region)}

In this subsection our aim is to quantify the impact of channel dynamics on the extent of the transitional region. Given that the channel model is a function of SNR versus distance and the receiver response is a function of PRR versus SNR, we can derive the behavior of PRR versus distance by linking both expressions through the SNR metric. First, we derive the SNR values that determine the beginning and end of the transitional region in the receiver response, and then we determine how these SNR values map to distance in the channel model.

Even though there are no strict definitions for the different regions in the literature, two valid definitions the following are.

Definition 1. In the connected region, links have a high probability $\left(>p_{h}\right)$ of having high packet reception rates $\left(>\psi_{h}\right)$.

Definition 2. In the disconnected region, links have a high probability $\left(>p_{\ell}\right)$ of having low packet reception rates $\left(<\psi_{\ell}\right)$ where $p_{h}$ and $p_{\ell}$ can be chosen as any numbers close to 1 and 0 , respectively.

Letting $\mathcal{B}_{M}^{-1}$.) be the inverse ${ }^{4}$ of $\mathcal{B}_{M}\left(\gamma_{d B}\right)$, and $\Psi^{-1}(\psi)=\mathcal{B}_{M}^{-1}\left(1-\psi^{1 / f}\right)$ the inverse of $\Psi$, the PRR values $\psi_{h}$ and $\psi_{\ell}$ from the preceding definitions can be mapped to their corresponding SNR values in dB: $\gamma_{h}=\Psi^{-1}\left(\psi_{h}\right)$ and $\gamma_{\ell}=$ $\Psi^{-1}\left(\psi_{\ell}\right)$. These SNR values determine the beginning and end of the transitional region.

Figure 1(a) shows how $\psi_{h}$ and $\psi_{\ell}$ determine three different regions in the radio-receiver response (Eq. (5)), and Figure 1(b) shows how $\gamma_{h}$ and $\gamma_{\ell}$ interact with the channel (Eq. (3)) to determine the extent of the connected, transitional, and disconnected regions.

According to Definition 1 , the beginning of the transitional region $\left(d_{b}\right)$ satisfies the following condition:

$$
\begin{aligned}
& p\left(\Psi>\psi_{h}\right) \quad=p_{h}, \because \Psi \text { is injective } \\
& \Rightarrow p\left(\Upsilon>\gamma_{h}\right) \quad=p_{h}, \because \Upsilon \text { is Gaussian } \\
& \Rightarrow Q\left(\frac{\gamma_{h}-\mu\left(d_{b}\right)}{\sigma}\right)=p_{h}
\end{aligned}
$$

\footnotetext{
${ }^{4}$ BER functions are injective. Hence, while there might not be a closed-form expression for their inverse function, the SNR in the domain can always be obtained numerically.
} 


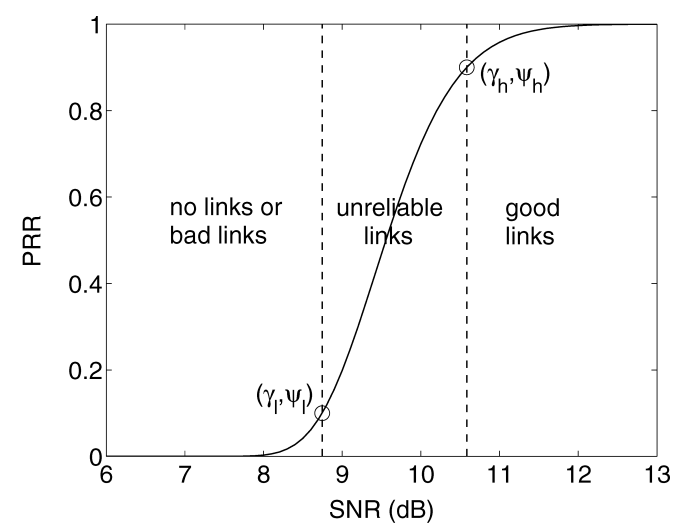

(a)

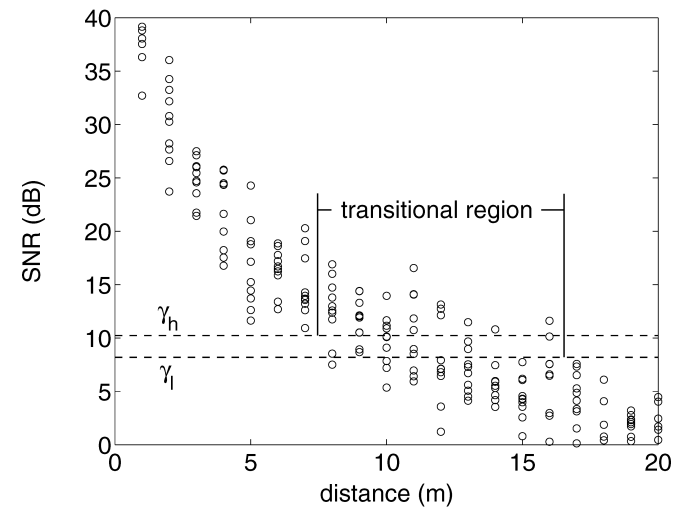

(b)

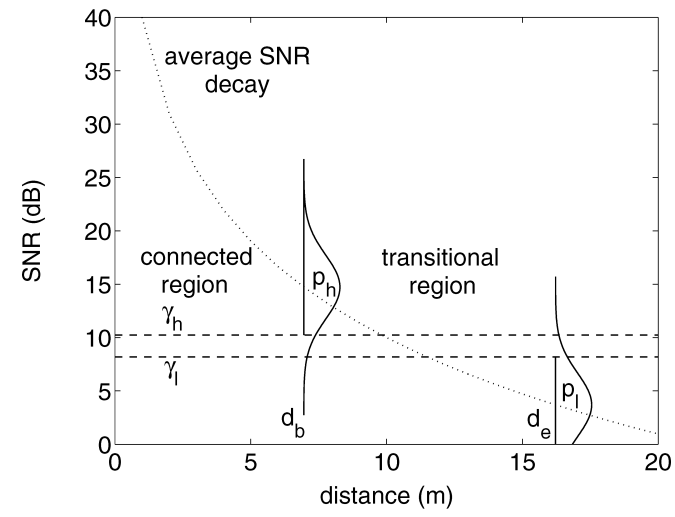

(c)

Fig. 1. (a) A receiver response where $\psi_{\ell}$ and $\psi_{h}$ determine different regions of link quality; (b) interaction of $\gamma_{\ell}$ and $\gamma_{h}$ with the channel to determine the transitional region; (c) analytical representation of Eq. (6) and (7). 
Moreover, according to Definition 2, the end of transitional region $\left(d_{e}\right)$ satifies

$$
\begin{aligned}
& p\left(\Psi<\psi_{\ell}\right) \quad=p_{\ell}, \quad \because \Psi \text { is injective } \\
& \Rightarrow p\left(\Upsilon<\gamma_{\ell}\right) \quad=p_{\ell}, \\
& \Rightarrow p\left(\Upsilon \geq \gamma_{\ell}\right) \quad=\left(1-p_{\ell}\right), \because \Upsilon \text { is Gaussian } \\
& \Rightarrow Q\left(\frac{\gamma_{\ell}-\mu\left(d_{e}\right)}{\sigma}\right)=\left(1-p_{\ell}\right),
\end{aligned}
$$

where $Q($.$) is the tail integral of a unit Gaussian probability density function$ $(p d f)$ and $\mu($.$) is given by Eq. (4). Figure 1(c) depicts an analytical representation$ of the previous equations. The figure shows how the interaction between the channel and receiver response determines the extent of the transitional region. Finally, $d_{b}$ and $d_{e}$ can be derived from Eq. (6) and (7)

$$
\begin{gathered}
d_{b}=10^{\frac{\gamma_{h}-\sigma Q^{-1}\left(p_{h}\right)-P_{t}+P_{n}+P L\left(d_{0}\right)}{-10 n}} \\
d_{e}=10^{\frac{\gamma \gamma^{-\sigma Q} Q^{-1}\left(1-p_{\ell}\right)-P_{t}+P_{n}+P L\left(d_{0}\right)}{-10 n}}
\end{gathered}
$$

while Eq. (8) provides absolute values for the extent of the different regions, it may not be useful to compare the link qualities of different scenarios. With that aim, we define the transitional region coefficient $\Gamma$, which is the ratio of the extent of the transitional with respect to the extent of the connected region.

$$
\begin{aligned}
\Gamma & =\frac{d_{e}-d_{b}}{d_{b}} \\
& =10^{\frac{\left(y_{h}-\gamma_{\ell}\right)+\sigma\left(Q^{-1}\left(1-p_{\ell}\right)-Q^{-1}\left(p_{h}\right)\right)}{10 n}}-1
\end{aligned}
$$

The lower the coefficient $\Gamma$, the smaller the transitional region compared to the connected one. For example, for the ideal binary model where $\gamma_{h}=\gamma_{\ell}$ and $\sigma=0$, the coefficient $\Gamma=0$. Notice that $\Gamma$ is independent of the noise floor $P_{n}$ and output power $P_{t}$; a higher output power would increase the connected region, but would increase the transitional region as well, keeping a constant ratio.

Eq. (9) predicts the impact of the channel on the transitional region. Given that $p_{h}$ and $p_{\ell}$ are high probabilities, $\left(Q^{-1}\left(1-p_{\ell}\right)-Q^{-1}\left(p_{h}\right)\right)$ is positive and hence, while a small $\sigma$ decreases the relative extent of the transitional region, a small $\eta$ increases it. Therefore, scenarios with high $\eta$ and low $\sigma$ reduce the relative size of the transitional region. Figure 2 (a) presents $\Gamma$ for different values of $\eta$ and $\sigma$, where $p_{\ell}=p_{h}=0.9, \gamma_{h}=10.23 \mathrm{~dB}$, and $\gamma_{\ell}=8.20 \mathrm{~dB}{ }^{5}$

Figure 2(b) depicts analytically the impact of $\eta$ and $\sigma$ on the extent of the transitional region. The SNR bounds on the radio receiver $\left(\gamma_{h}\right.$ and $\left.\gamma_{\ell}\right)$ are fixed and independent of environment. When $\sigma$ increases from 1 to 2 , the signal values ( $y$ axis) have a higher probability of entering the transitional region more closely to the transmitter and leaving it at farther distances, resulting in a larger transitional region. When $\eta$ is increased (left arrow), the faster decay of the signal strength decreases the width of the transitional region.

\footnotetext{
${ }^{5} \gamma_{h}$ and $\gamma_{\ell}$ were obtained for an NCFSK radio with Manchester encoding and a frame size of 100 bytes. Different modulations, encodings, and packet sizes do not have a significant impact on $\Gamma$, and the results are not presented due to space constraints. Some of these results are available in Zuniga and Krishnamachari [2004].
} 


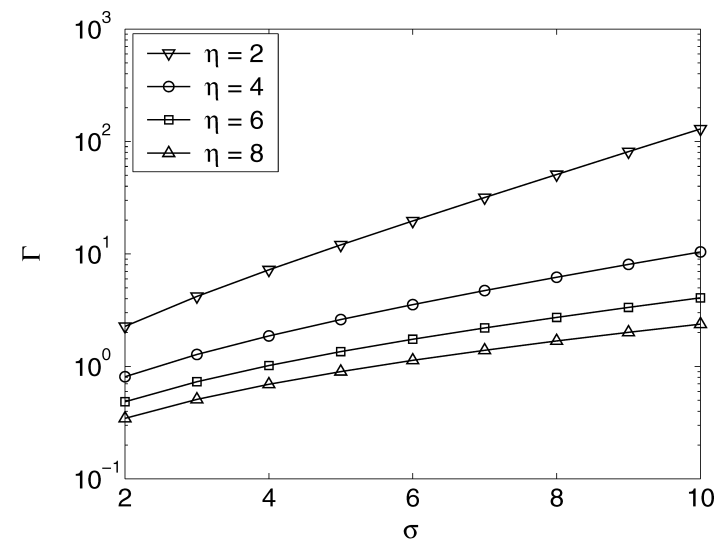

(a)

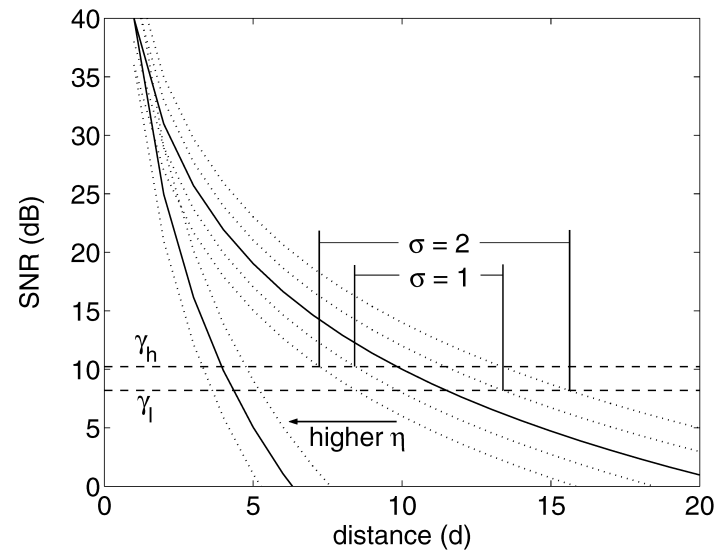

(b)

Fig. 2. Impact of $\sigma$ and $\eta$ : (a) on extent of transitional region; (b) $\Gamma$ for different values of $\eta$ and $\sigma$. Solid curves represent average power decay, and dotted lines the $[-2 \sigma, 2 \sigma]$ interval of the variance.

Eq. (9) also predicts the impact of the receiver. The sharper the receiver threshold, the smaller $\left(\gamma_{h}-\gamma_{\ell}\right)$ and the smaller the $\Gamma$ coefficient. However, even with a perfect threshold receiver $\left(\gamma_{h}=\gamma_{\ell}\right)$, such as that used on the ideal model, the transitional region would still exist due to channel dynamics $(\sigma)$. Figure 3(a) depicts analytically the behavior of a perfect threshold receiver in a real channel, and Figure 3(b) shows an instance of link behavior. Notice that in this hypothetical scenario, the transitional region would consist only of 0/1 links.

The model also allows providing the cumulative distribution function (cdf) of the packet reception rate as a function of distance. According to Eq. (5),

$$
\begin{aligned}
F(\psi) & =p(\Psi<\psi) \\
& =p\left(\Upsilon<\Psi^{-1}(\psi)\right) \\
& =1-Q\left(\frac{\Psi^{-1}(\psi)-\mu(d)}{\sigma}\right),
\end{aligned}
$$




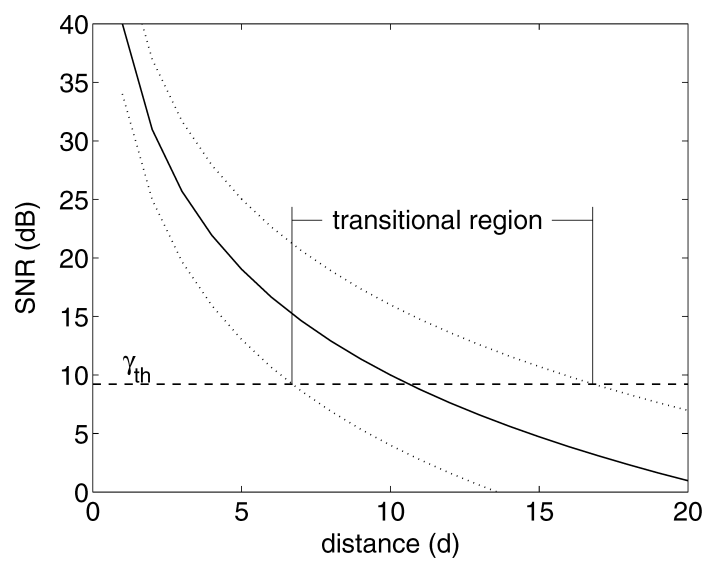

(a)

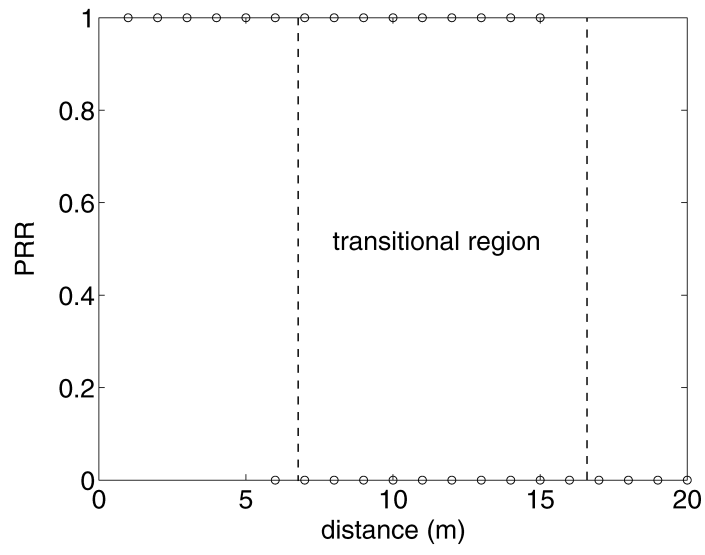

(b)

Fig. 3. Impact of perfect receiver threshold on extent of transitional region; (a) analytical representation; (b) an instance of PRR vs. distance.

where $\mu(d)$ is the average SNR decay (Eq. (4)). Figure 4 shows an example of the cumulative distribution $F(\psi)$ for $\eta=3$ and $\sigma=3$. Three different transmitter-receiver distances are shown: the end of the connected, middle of the transitional, and beginning of the disconnected region. We can notice that independent of the region where the receiver is, the link has a higher probability of being either good or bad (above 0.9 or below 0.1 PRR) than of being unreliable (between 0.9 and 0.1 ). For instance, in the middle of the transitional region the link has a $30 \%$ probability of being unreliable; and the probability of observing unreliable links at the end of the connected region or beginning of the disconnected is small $(<5 \%)$. Empirical measurements in Cerpa et al. [2005] and Zuniga and Krishnamachari [2004] agree with the analytical $c d f$ in Eq. (10).

We remark that the obtained $c d f$ s are valid only for the scope of this work (static and low-dynamic environments); highly dynamic environments add a new dimension of time to the $c d f$ s. 


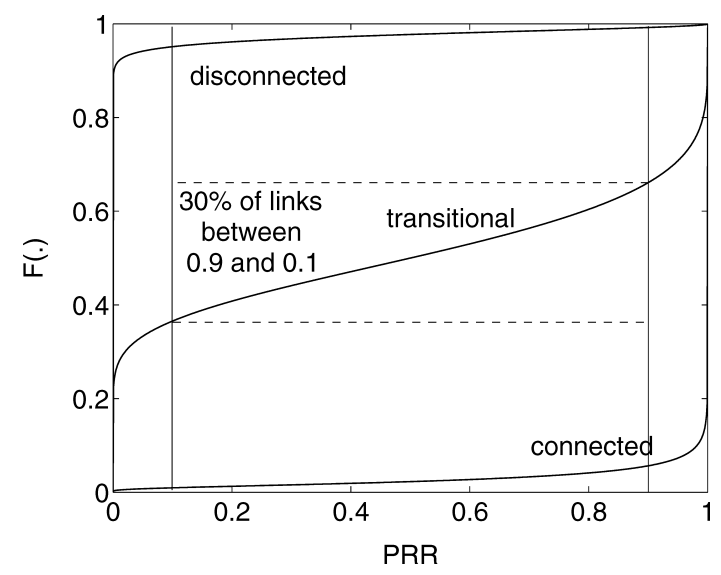

Fig. 4. The $c d f \mathrm{~s}$ for packet reception rate for receivers in different regions in a specific environment $(\eta=3, \sigma=3)$.

\subsection{Expectation and Variance of Packet Reception Rate}

Even though longer distance does not necessarily imply lower packet reception rate, the expected value of the packet reception rate does decrease monotonically with distance in a given propagation direction. ${ }^{6}$ In this subsection, we present approximate expressions for the expectation and variance of the packet reception rate $\Psi$. These expressions are important because they confirm mathematically that the transitional region has a higher variability in PRR than the connected region.

First we present general expressions for the expectation and variance in Eqs. (11) and (12). These expressions depend on the PRR versus distance (receiver response in Eq. (5)) and the probability density functions ( $p d f$ ) of the SNR for a given distance $d$ (which is log-normally distributed). Given the mathematical complexity of dealing with the receiver response and $p d f$, we present linear approximations for both that are used to derive approximate expressions for the expectation and variance of the packet reception rate $\Psi$.

In general, the first two moments of $\Psi$ are defined by

$$
\begin{gathered}
E[\Psi]=\int_{-\infty}^{\infty} \Psi\left(\gamma_{d B}\right) f\left(\gamma_{d B}, d\right) \delta \gamma_{d B} \\
E\left[\Psi^{2}\right]=\int_{-\infty}^{\infty} \Psi^{2}\left(\gamma_{d B}\right) f\left(\gamma_{d B}, d\right) \delta \gamma_{d B},
\end{gathered}
$$

where $f\left(\gamma_{d B}, d\right)$ represents the $p d f$ of SNR (a Gaussian random variable with parameters $\mu(d)$ and $\sigma)$.

\footnotetext{
${ }^{6}$ The radio model used in this work is isotropic, but this is not true of practical antennas. By linearity of expectation, since $E\left[\Psi_{a}(d)\right]$ is monotonic with distance for a given propagation direction $a$, it can be shown that the expected PRR averaged over all angles is also monotonic with distance; however, it should be kept in mind that expected PRR values at different angles may show nondistancemonotonic behavior with respect to each other.
} 


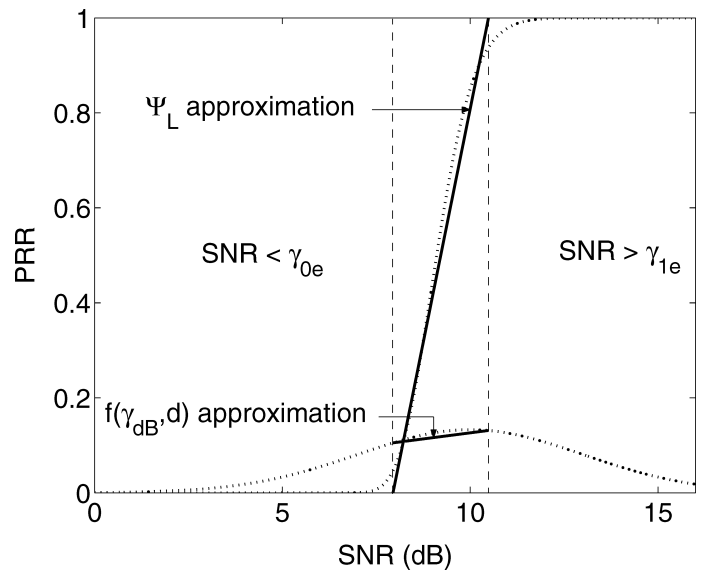

Fig. 5. Linear approximation of receiver threshold and Gaussian SNR; the mean of the Gaussian depends on the transmitter-receiver distance.

The sharp thresholds of $\Psi$ and $\Psi^{2}$ permit the linear approximations

$$
\begin{aligned}
& \Psi(\gamma) \approx \Psi_{L}(\gamma)= \begin{cases}0, & \gamma \leq \gamma_{0 e} \\
m_{e} \gamma+b_{e}, & \gamma_{0 e}<\gamma<\gamma_{1 e} \\
1, & \gamma \geq \gamma_{1 e}\end{cases} \\
& \Psi^{2}(\gamma) \approx \Psi_{L}^{2}(\gamma)= \begin{cases}0, & \gamma \leq \gamma_{0 v} \\
m_{v} \gamma+b_{v}, & \gamma_{0 v}<\gamma<\gamma_{1 v} \\
1, & \gamma \geq \gamma_{1 v},\end{cases}
\end{aligned}
$$

where $m_{e}, m_{v}$ and $b_{e}, b_{v}$ are the slopes and $y$-intercepts of the linear approximations $\Psi_{L}$ and $\Psi_{L}^{2}$, and $\gamma$ is in dB. Figure 5(a) shows the approximation procedure for $\Psi_{L}$; the procedure for $\Psi_{L}^{2}$ is similar. The mechanism to obtain the slopes, $y$ intercepts, and limit points of Eqs. (13) and (14) is presented later.

The linear models lead to the following approximations of Eqs. (11) and (12):

$$
\begin{aligned}
E[\Psi] \approx & \int_{\gamma_{0 e}}^{\infty} \Psi_{L}\left(\gamma_{d B}\right) f\left(\gamma_{d B}, d\right) \delta \gamma_{d B} \\
= & \int_{\gamma_{0 e}}^{\gamma_{1 e}}\left(m_{e} \gamma+b_{e}\right) f\left(\gamma_{d B}, d\right) \delta \gamma_{d B} \\
& +Q\left(\frac{\gamma_{1 e}-\mu(d)}{\sigma}\right) \\
E\left[\Psi^{2}\right] \approx & \int_{\gamma_{0 v}}^{\infty} \Psi_{L}^{2}\left(\gamma_{d B}\right) f\left(\gamma_{d B}, d\right) \delta \gamma_{d B} \\
= & \int_{\gamma_{0 v}}^{\gamma_{1 v}}\left(m_{v} \gamma+b_{v}\right) f\left(\gamma_{d B}, d\right) \delta \gamma_{d B} \\
& +Q\left(\frac{\gamma_{1 v}-\mu(d)}{\sigma}\right)
\end{aligned}
$$


In the preceding approximations $f\left(\gamma_{d B}, d\right)$ is evaluated separately on intervals $\left[\gamma_{0 e}, \gamma_{1 e}\right]$ and $\left[\gamma_{0 v}, \gamma_{1 v}\right]$ for $E[\Psi]$ and $E\left[\Psi^{2}\right]$, respectively. Both intervals represent linear approximations of the sharp thresholds of $\Psi$ and $\Psi^{2}$, and these thresholds are narrow compared to the $[\mu-4 \sigma, \mu+4 \sigma]$ domain of $f\left(\gamma_{d B}, d\right),{ }^{7}$ hence, linear approximations can be used as well for $f\left(\gamma_{d B}, d\right)$ in $\left[\gamma_{0 e}, \gamma_{1 e}\right]$ and $\left[\gamma_{0 v}, \gamma_{1 v}\right]$.

Let us denote $f_{\Psi}\left(\gamma_{d B}, d\right)$ and $f_{\Psi^{2}}\left(\gamma_{d B}, d\right)$ as the linear approximations of $f\left(\gamma_{d B}, d\right)$ for intervals $\left[\gamma_{0 e}, \gamma_{1 e}\right]$ and $\left[\gamma_{0 v}, \gamma_{1 v}\right]$ :

$$
\begin{gathered}
f_{\Psi}\left(\gamma_{d B}, d\right)=m_{g e} \gamma+b_{g e} \\
f_{\Psi^{2}}\left(\gamma_{d B}, d\right)=m_{g v} \gamma+b_{g v}
\end{gathered}
$$

where

$$
\begin{aligned}
& m_{g e}=\frac{f\left(\gamma_{1 e}, d\right)-f\left(\gamma_{0 e}, d\right)}{\gamma_{1 e}-\gamma_{0 e}} \quad b_{g e}=\frac{f\left(\gamma_{0 e}, d\right) \gamma_{1 e}-f\left(\gamma_{1 e}, d\right) \gamma_{0 e}}{\gamma_{1 e}-\gamma_{0 e}} \\
& m_{g v}=\frac{f\left(\gamma_{1 v}, d\right)-f\left(\gamma_{0 v}, d\right)}{\gamma_{1 v}-\gamma_{0 v}} b_{g v}=\frac{f\left(\gamma_{0 v}, d\right) \gamma_{1 v}-f\left(\gamma_{1 v}, d\right) \gamma_{0 v}}{\gamma_{1 v}-\gamma_{0 v}} .
\end{aligned}
$$

Figure 5 shows the approximation procedure for $f_{\Psi}\left(\gamma_{d B}, d\right)$ (Gaussian SNR curve for $E[\Psi])$; the procedure for $f_{\Psi^{2}}\left(\gamma_{d B}, d\right)$ is similar.

Finally, based on Eqs. (15) and (16), the first and second moment approximations of the packet reception rate are given by

$$
\begin{aligned}
E[\Psi] \approx & \int_{\gamma_{0 e}}^{\gamma_{1 e}}\left(m_{e} \gamma+b_{e}\right) f_{\Psi}\left(\gamma_{d B}, d\right) \delta \gamma_{d B} \\
& +Q\left(\frac{\gamma_{1 e}-\mu(d)}{\sigma}\right) \\
= & \int_{\gamma_{0 e}}^{\gamma_{1 e}}\left(m_{e} \gamma+b_{e}\right)\left(m_{g e} \gamma+b_{g e}\right) \delta \gamma_{d B} \\
& +Q\left(\frac{\gamma_{1 e}-\mu(d)}{\sigma}\right) \\
= & \left(m_{e} \times m_{g e}\right) \frac{\gamma^{3}}{3}+\left(b_{e} m_{g e}+b_{g e} m_{e}\right) \frac{\gamma^{2}}{2} \\
& \left.+b_{e} b_{g e} \gamma\right)\left.\right|_{\gamma_{0 e}} ^{\gamma_{1 e}}+Q\left(\frac{\gamma_{1 e}-\mu(d)}{\sigma}\right) \\
E\left[\Psi^{2}\right] \approx & \int_{\gamma_{0 v}}^{\gamma_{1 v}}\left(m_{v} \gamma+b_{v}\right) f_{\Psi^{2}}\left(\gamma_{d B}, d\right) \delta \gamma_{d B} \\
& +Q\left(\frac{\gamma_{1 v}-\mu(d)}{\sigma}\right)
\end{aligned}
$$

\footnotetext{
${ }^{7}$ While the domain of a Gaussian random variable is $[-\infty,+\infty]$, the interval $[\mu-4 \sigma, \mu+4 \sigma]$ contains most of the probability space (.999), and this is wide compared to the sharp threshold of the receiver for common values of $\sigma$ [Sohrabi et al. 1999].
} 


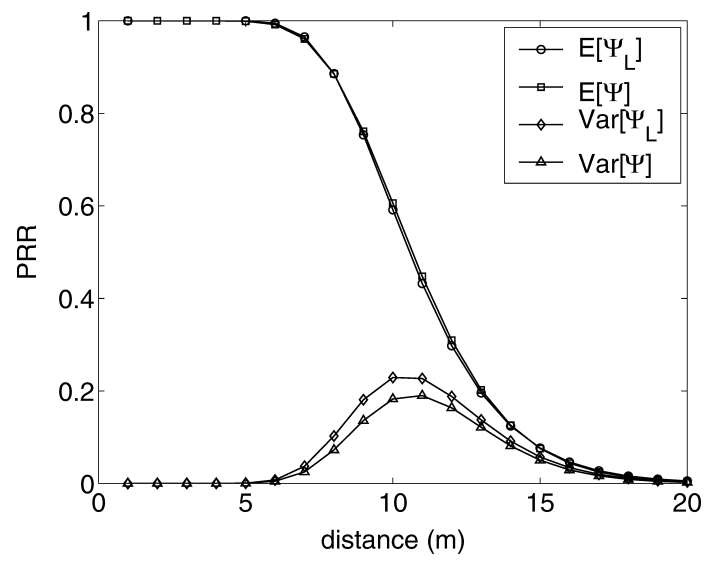

Fig. 6. Comparison of $\mathrm{E}[\Psi]$ and $\operatorname{Var}[\Psi]$ with their linear approximations $\mathrm{E}\left[\Psi_{L}\right]$ and $\operatorname{Var}\left[\Psi_{L}\right]$.

$$
\begin{aligned}
= & \int_{\gamma_{0 v}}^{\gamma_{1 v}}\left(m_{v} \gamma+b_{v}\right)\left(m_{g v} \gamma+b_{g v}\right) \delta \gamma_{d B} \\
& +Q\left(\frac{\gamma_{1 v}-\mu(d)}{\sigma}\right) \\
= & \left(\left(m_{v} \times m_{g v}\right) \frac{\gamma^{3}}{3}+\left(b_{v} m_{g v}+b_{g v} m_{v}\right) \frac{\gamma^{2}}{2}\right. \\
& \left.+b_{v} b_{g v} \gamma\right)\left.\right|_{\gamma_{0 v}} ^{\gamma_{1 v}}+Q\left(\frac{\gamma_{1 v}-\mu(d)}{\sigma}\right) .
\end{aligned}
$$

In general, the parameters of $\Psi_{L}$ and $\Psi_{L}^{2}$ (slopes, $y$-intercepts, and limit points of Eqs. (13) and (14)) can be obtained by curve-fitting $\Psi$ and $\Psi^{2}$ through least squares regression techniques. Nevertheless, our studies suggest that choosing a line that passes through points $\mathrm{A}$ and $\mathrm{B}$ with PRRs of 0.1 and 0.9 provides an accurate approximation. ${ }^{8}$ Hence, $A$ and $B$ defined as $\left(\Psi^{-1}(0.1), 0.1\right)$ and $\left(\Psi^{-1}(0.9), 0.9\right)$ can be used to obtain the different parameters of $\Psi_{L}$ :

$$
\begin{aligned}
& m_{e}=\frac{0.9-0.1}{\gamma_{B}-\gamma_{A}} \quad b_{e}=\frac{0.1 \gamma_{B}-0.9 \gamma_{A}}{\gamma_{B}-\gamma_{A}} \\
& \gamma_{0 v}=\frac{-b_{e}}{m_{e}} \quad \gamma_{1 v}=\frac{1-b_{e}}{m_{e}},
\end{aligned}
$$

where $\gamma_{A}=\Psi^{-1}(0.1)$ and $\gamma_{B}=\Psi^{-1}(0.9)$, both in $\mathrm{dB}$. For $\Psi_{L}^{2}$, points A and B are $\left(\Psi^{-1}(\sqrt{0.1}), 0.1\right)$ and $\left(\Psi^{-1}(\sqrt{0.9}), 0.9\right)$.

Figure 6 shows an example of numerically calculated curves for the expectation and variance (from Eqs. (11) and (12)), and their approximations through Eqs. (19) and (20) for $\eta=3$ and $\sigma=3$. In general, the error depends on the parameters of $f\left(\gamma_{d B}, d\right)$ (i.e., the $p d f$ of SNR). The smaller the $\sigma$, the larger the error because the width of the receiver threshold starts to be comparable

${ }^{8}$ Actually, no significant differences were found if points A and B are chosen in intervals [0.01, 0.2] and $[0.8,0.99]$, respectively. 


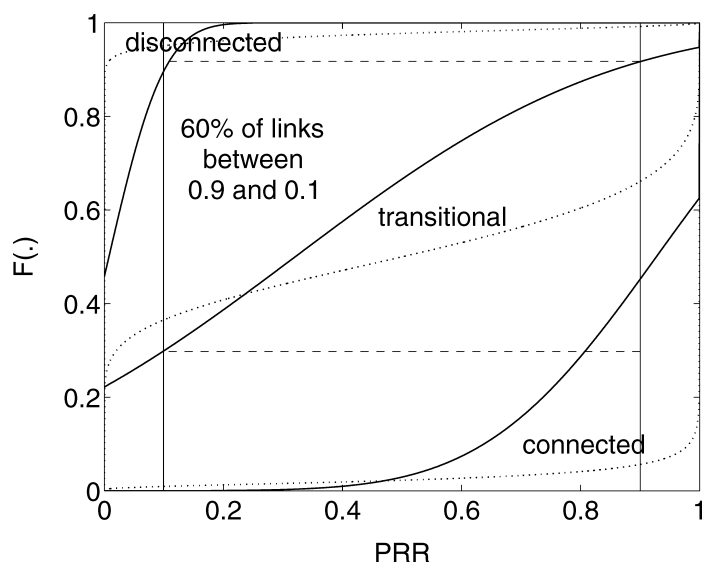

Fig. 7. Comparison of $c d f \mathrm{~s}$ between the Gaussian model (black curves) and our analytical model (dotted curves) for receivers in different regions.

with the width of the bell of the Gaussian curve, which leads to less accurate linearization. However, for common values of $\sigma$ [Sohrabi et al. 1999], the bell is significantly wider than the receiver threshold and the approximation errors are not significant. Also, while the expectation decreases monotonically with distance, the variance has a bell shape whose maximum lies in the transitional region; this behavior agrees with empirical observations in Woo et al. [2003].

\subsection{Comparison With Available Link Models}

Some popular wireless network simulators [Levis et al. 2003; Girod et al. 2004] and recent studies [Woo et al. 2003] have been using a Gaussian random variable to represent the packet reception rate. The PRR function based on the Gaussian model $\left(\Psi_{G}\right)$ has the following form:

$$
\Psi_{G}= \begin{cases}1, & X>1 \\ x, & 0 \leq X \leq 1 \\ 0, & X<0,\end{cases}
$$

where $X$ is a Gaussian random variable with parameters $\mu=E[\Psi]$ and $\sigma^{2}=$ $\operatorname{Var}(\Psi)$. The Gaussian model leads to the following $c d f F_{G}$ :

$$
F_{G}(\psi)= \begin{cases}1-Q\left(\frac{-E[\Psi]}{\sqrt{V[\Psi]}}\right), & \psi=0 \\ 1-Q\left(\frac{\psi-E[\Psi]}{\sqrt{V[\Psi]}}\right), & 0<\psi<1 \\ 1, & \psi=1\end{cases}
$$

Figure 7 shows a comparison between the $c d f$ s of the Gaussian model (Eq. (22)) and our analytical model (Eq. (10)) for receivers in connected, transitional, and disconnected regions. Contrary to the analytical $c d f$ where links have higher probability of being either good or bad (above 0.9 or below 0.1 PRR), the 
Gaussian model leads to links that have a high probability of being between 0.9 and 0.1 ; or $60 \%$ for the transitional region and $40 \%$ for the connected region, which may lead to misleading results in protocol testing. The results shown are for $\eta=3, \sigma=3$, and a noncoherent FSK radio, but similar trends are obtained for different parameters.

\section{IMPACT OF HARDWARE VARIANCE}

In the previous section it was assumed that all radios have the same output power $P_{t}$ and noise floor $P_{n}$, however, hardware variance induces some fluctuation around the output power that is set by the user and around the average noise floor. This variance problem is partially solved during the manufacturing process, where radios with a low output power and/or high noise floor (low sensitivity) are usually discarded. However, no upper bound is used in the filtering process and hardware variance remains a problem. As stated in Poor [2004]: This filtering process is justifiable, since radios that are more powerful or more sensitive are generally desirable.

Hardware variance has already been identified as the cause of asymmetric links [Ganesan et al. 2003]. In this section, we not only quantify the effect of hardware variance on link asymmetry, but also show that hardware variance can have a significant impact on the extent of the transitional region.

It is important to notice that while the output power variance can be calibrated to the same value for all radios, the noise floor variance cannot be eliminated through calibration, since it depends on the thermal noise generated by the underlying solid-state structure.

\subsection{Model}

Hardware variance causes Gaussian distributions (in $\mathrm{dB}$ ) in the output power and noise floor [Poor 2004]. In order to capture these effects, let us redefine Eq. (3) by denoting $S N R_{A B}$ as the signal-to-noise ratio measured at $\mathrm{B}$ for the output power of $\mathrm{A}$. Then $S N R_{A B}\left(\Upsilon_{A B}\right)$ is given by

$$
\begin{aligned}
\Upsilon_{A B} & =P_{t A}-P L(d)-P_{n B} \\
& =\mathcal{N}\left(P_{t}, \sigma_{t x}\right)-P L(d)-\mathcal{N}\left(P_{n}, \sigma_{r x}\right),
\end{aligned}
$$

where $\sigma_{t x}^{2}$ are $\sigma_{r x}^{2}$ are the variances of the output power the noise floor, respectively, and $P L(d)=P L\left(d_{0}\right)+10 \eta \log _{10}\left(\frac{d}{d_{0}}\right)+\mathcal{N}(0, \sigma)$ is the channel path loss (which is identical in both directions: $A \rightarrow B$ and $B \rightarrow A$ ).

Empirical measurements (see Section 5) show that there is some correlation between the output power and noise floor within the same radio. Our model captures this correlation by representing the output power and noise floor as a multivariate Gaussian distribution, as shown next:

$$
\left(\begin{array}{c}
T \\
R
\end{array}\right) \sim \mathcal{N}\left(\left(\begin{array}{c}
P_{t} \\
P_{n}
\end{array}\right),\left(\begin{array}{cc}
S_{T} & S_{T R} \\
S_{R T} & S_{R}
\end{array}\right)\right)
$$

where $P_{t}$ is the nominal output power, $P_{n}$ the average noise floor, $S$ the covariance matrix between the output power and noise floor, and $T$ and $R$ the actual output power and noise floor of a specific radio, respectively. 


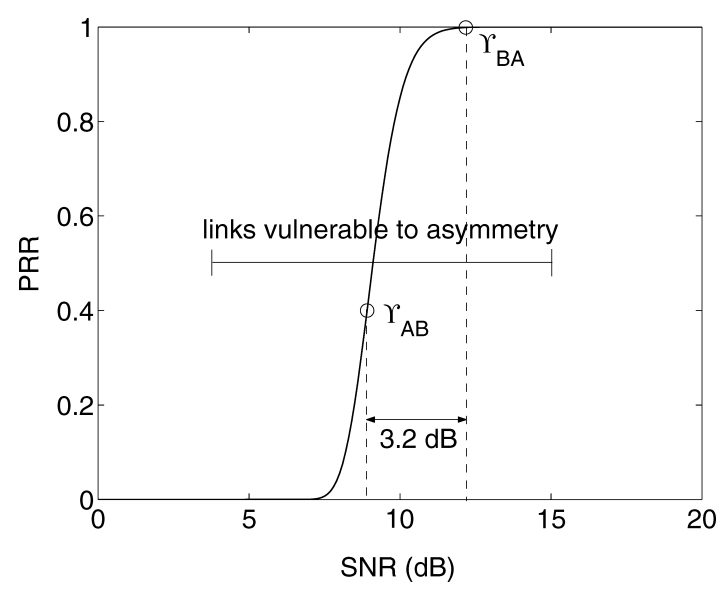

Fig. 8. Impact of hardware variance on asymmetric links.

\subsection{Impact on Asymmetric Links}

When the output power level of all nodes is set to the same value, radios with identical nonvariant hardware $\left(\sigma_{t x}=0, \sigma_{r x}=0\right)$ lead to the same SNR in both directions $\left(\Upsilon_{A B}=\Upsilon_{B A}\right.$ according to Eq. (23)), which in turn leads to the same packet reception rate (i.e., symmetric links).

For radios with hardware variance, $\Upsilon_{A B}$ can differ from $\Upsilon_{B A}$. Figure 8 shows the effect of $\Upsilon_{A B}-\Upsilon_{B A}$ on link asymmetry. Due to the sharp threshold of the receiver, a small value of $\Upsilon_{A B}-\Upsilon_{B A}(\sim 3.2 \mathrm{~dB})$ may lead to significantly different packet reception rates in both directions (1.0 and 0.4).

$\Upsilon_{A B}-\Upsilon_{B A}$ is a random variable and the larger the variance of this difference, the higher the probability of link asymmetry. In order to quantify the impact of hardware variance on link asymmetry we will analyze the variance of $\left(\Upsilon_{A B}-\right.$ $\left.\Upsilon_{B A}\right)$.

Letting $\left(T_{A}, R_{A}\right)$ and $\left(T_{B}, R_{B}\right)$ be the respective output power and noise floor of radios $\mathrm{A}$ and $\mathrm{B}$, then

$$
\begin{aligned}
\Upsilon_{A B}-\Upsilon_{B A}= & \left(T_{A}-P L(d)-R_{B}\right) \\
& -\left(T_{B}-P L(d)-R_{A}\right) \\
= & \left(T_{A}+R_{A}\right)-\left(T_{B}+R_{B}\right) .
\end{aligned}
$$

Here, $\left(T_{A}+R_{A}\right)$ and $\left(T_{B}+R_{B}\right)$ are Gaussian random variables representing the sum of the output power and noise floor of different radios $(A$ and $B)$, and can be assumed independent. ${ }^{9}$ Moreover, $\left(T_{A}+R_{A}\right)$ and $\left(T_{B}+R_{B}\right)$ are generated from the same multivariate Gaussian distribution and can be represented by

\footnotetext{
${ }^{9}$ The manufacturing process can create some correlation among different radios if different batches are produced from special high- (low-) quality materials, but we assume that all radios belong to the same process.
} 
$(T+R)$, hence $\operatorname{Var}\left(\Upsilon_{A B}-\Upsilon_{B A}\right)=2 \times \operatorname{Var}(T+R),{ }^{10}$ and

$$
\begin{aligned}
\operatorname{Var}(T+R)= & E\left[(T+R)^{2}\right]-E^{2}[T+R] \\
= & E\left[T^{2}\right]-E^{2}[T]+E\left[R^{2}\right]-E^{2}[R] \\
& +2(E[T R]-E[T] E[R]) \\
= & \operatorname{Var}(T)+\operatorname{Var}(R)+2 \operatorname{Cov}(T, R) \\
= & S_{T}+S_{R}+2 S_{T R},
\end{aligned}
$$

which leads to

$$
\operatorname{Var}\left(\Upsilon_{A B}-\Upsilon_{B A}\right)=2\left(S_{T}+S_{R}+2 S_{T R}\right),
$$

where $S_{T}, S_{R}$, and $S_{T R}$ are elements of the covariance matrix in Eq. (24).

Eq. (27) shows that a positive correlation (i.e., positive $S_{T R}$ ) between the output power and noise floor of a radio leads to high variance of $\Upsilon_{A B}-\Upsilon_{B A}$ (i.e., higher probability of link asymmetry), while a negative correlation (negative $S_{T R}$ ) reduces the variance (lower probability of link asymmetry). Hence, a negative correlation between the output power and noise floor leads to the lowest probability of link asymmetry, followed by zero correlation and positive correlation. Notice that a negative correlation implies that nodes with output powers higher than $P_{t}$ (i.e., better transmitter) will usually have a noise floor lower than $P_{n}$ (better receiver), and vice versa.

\subsection{Impact on Extent of Transitional Region}

In Eq. (3), the randomness of the SNR was due uniquely to multipath effects, but the variance of the output power and noise floor introduces two other sources of randomness. The combined effect of output power variance, channel multipaths, and noise floor variance leads to a new expression for the SNR (Eq. (23)). Based on this equation the $\mathrm{SNR} \Upsilon$ is given by

$$
\begin{aligned}
\Upsilon & =\mathcal{N}\left(P_{t}, \sigma_{t x}\right)-P L(d)-\mathcal{N}\left(P_{n}, \sigma_{r x}\right) \\
& =\mathcal{N}\left(P_{t}-P_{n}, \sigma_{h w}\right)-P L(d),
\end{aligned}
$$

where $\sigma_{h w}^{2}=\sigma_{t x}^{2}+\sigma_{r x}^{2}$. Finally, $\Upsilon$ is given by

$$
\begin{aligned}
\Upsilon & =\mathcal{N}\left(P_{t}-P_{n}, \sigma_{h w}\right)-\overline{P L\left(d_{0}\right)}+\mathcal{N}\left(0, \sigma_{c h}\right) \\
& =\mathcal{N}\left(P_{t}-\overline{P L\left(d_{0}\right)}-P_{n}, \sigma_{t}\right),
\end{aligned}
$$

where $\overline{P L\left(d_{0}\right)}=P L\left(d_{0}\right)+10 \eta \log _{10}\left(\frac{d}{d_{0}}\right)$, and the total variance of the system $\left(\sigma_{t}\right)$ is given by

$$
\begin{aligned}
\sigma_{t}^{2} & =\sigma_{c h}^{2}+\sigma_{t x}^{2}+\sigma_{r x}^{2} \\
& =\sigma_{c h}^{2}+\sigma_{h w}^{2},
\end{aligned}
$$

Hardware variance generates a pseudopath loss variance $\left(\sigma_{h w}\right)$. Eq. (9) shows that the larger the variance, the larger the extent of the transitional region;

\footnotetext{
${ }^{10}$ This is derived from the facts that for a random variable $X, \operatorname{Var}(X)=\operatorname{Var}(-X)$; and for i.i.d random variables $X_{i}, \operatorname{Var}\left(\sum_{i} X_{i}\right)=\sum_{i} \operatorname{Var}\left(X_{i}\right)$.
}

ACM Transactions on Sensor Networks, Vol. 3, No. 2, Article 7, Publication date: June 2007. 
hence, radios with hardware variance will always increase the extent of the transitional region.

To obtain accurate results for the extent of the transitional region, $\sigma$ should be replaced by $\sigma_{t}$ in all corresponding equations in Section 3. The impact of hardware variance on the extent of the transitional region can be observed in Figure 9, which presents simulated link qualities for $\eta=3, \sigma_{c h}=3$, and $\sigma_{h w}=3$. Figure 9(a) shows the transitional region when invariant hardware is placed in a real channel (i.e., effect of $\sigma_{c h}^{2}$ ) and Figure 9(b) presents a hypothetical scenario where variant hardware is placed in an ideal scenario (i.e., no multipath effects). We observe that even in the absence of multipath effects, a transitional region is observed due to the pseudovariance $\sigma_{h w}^{2}$. Finally, Figure 9(c) presents the combined effects of $\sigma_{c h}$ and $\sigma_{h w}$, showing a larger transitional region than in Figures 9(a) and (b).

\section{EMPIRICAL VALIDATION}

We now present empirical results conducted in static and low-dynamic environments to validate our analytical results on the impact of $\eta, \sigma_{c h}$, and $\sigma_{h w}$ on the extent of the transitional region. We will also observe that the correlation between output power and noise floor in mica2 motes is negative, which is the least damaging in terms of link asymmetry among the different correlations (positive, zero, negative).

We considered two environments: an indoor environment (aisle of a building), and an outdoor environment (football field). All measurements were made using mica2 motes. These devices use noncoherent FSK modulation at $915 \mathrm{MHz}$ with Manchester encoding and provide data rates of $38.4 \mathrm{Kbaud}$.

\subsection{Channel and Radio Parameters}

-Channel: Two motes were used to measure the path loss exponent $(\eta)$, variance $\left(\sigma_{c h}^{2}\right)$, and initial decay $P L\left(d_{0}\right)$ of the channel. Table III presents the values for $\eta$ and $\sigma_{c h}$. The reference distance $\left(d_{0}\right)$ of the log-normal model was set to $1 \mathrm{~m}$ and its corresponding power decay was found to be $55 \mathrm{~dB}$.

-Radio: One mote was selected as a common receiver and sender to capture the variance of the output power $P_{t}$ and noise floor $P_{n}$. The measurements were done in an isolated empty room, where each mote had the same source power and was placed at the same physical position with respect to the reference mote. Figure 10 presents the empirical measurements, which show a negative correlation between output power and noise floor. From our experiments, the resultant covariance matrix is given by

$$
S=\left(\begin{array}{cc}
6.0 & -3.3 \\
-3.3 & 3.7
\end{array}\right)
$$

The standard deviations of the output power $\left(\sigma_{t x}\right)$ and noise floor $\left(\sigma_{r x}\right)$ are presented in Table IV; these values lead to $\sigma_{h w}=3.0$. Different power levels were tested and all levels showed a similar variance.

The negative correlation of mica2 motes is due to several factors. Nowadays, chip implementation is moving toward single-chip design, hence, 


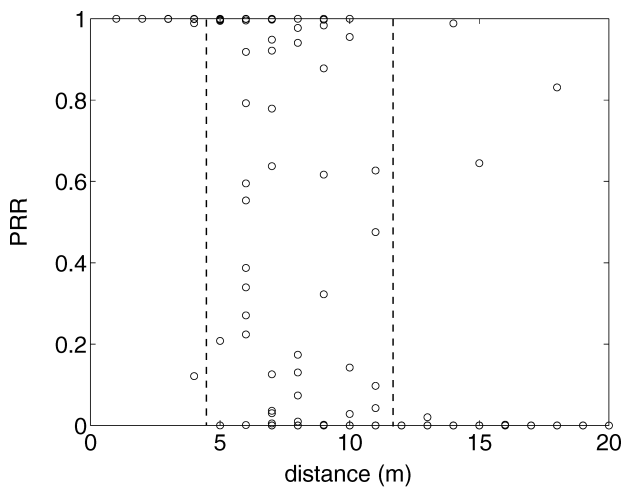

(a)

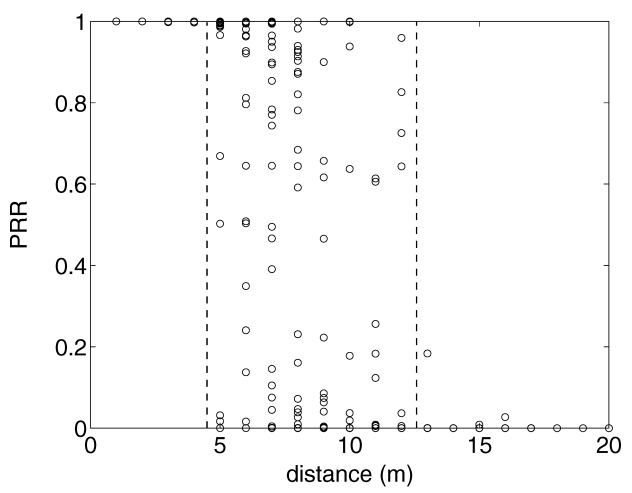

(b)

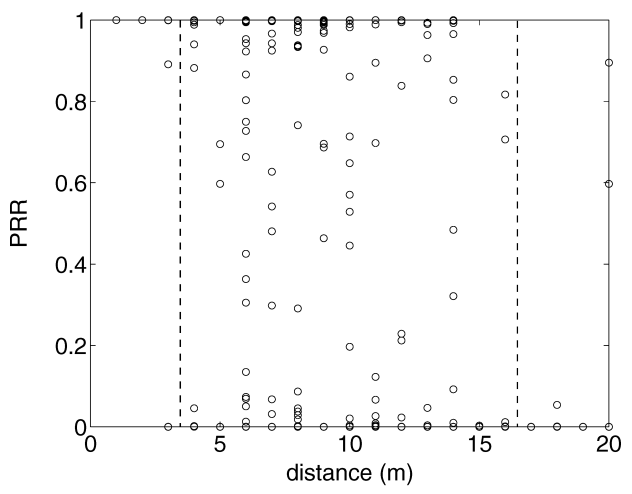

(c)

Fig. 9. Impact of channel and radio dynamics on extent of transitional region: (a) impact of channel dynamics (real channel + identical nonvariant hardware); (b) impact of hardware variance (ideal channel + hardware variance); (c) combined impact of channel and radio dynamics.

the performance of the transmitter and receiver is determined by the common underlying solid-state structure. Board implementation and antenna gains further enhance this correlation, since a common path goes from the antenna to the chip. Hence, a radio with a good solid-state structure (low thermal noise) 
Table III. Channel Parameters

\begin{tabular}{|l|c|c|}
\hline environment & $\eta$ (95\% conf. bounds) & $\sigma_{c h}(95 \%$ conf. bounds) \\
\hline outdoor & $4.7(4.3-5.1)$ & $3.2(2.6-3.8)$ \\
indoor & $3.3(2.1-4.5)$ & $5.5(4.6-6.8)$ \\
\hline
\end{tabular}

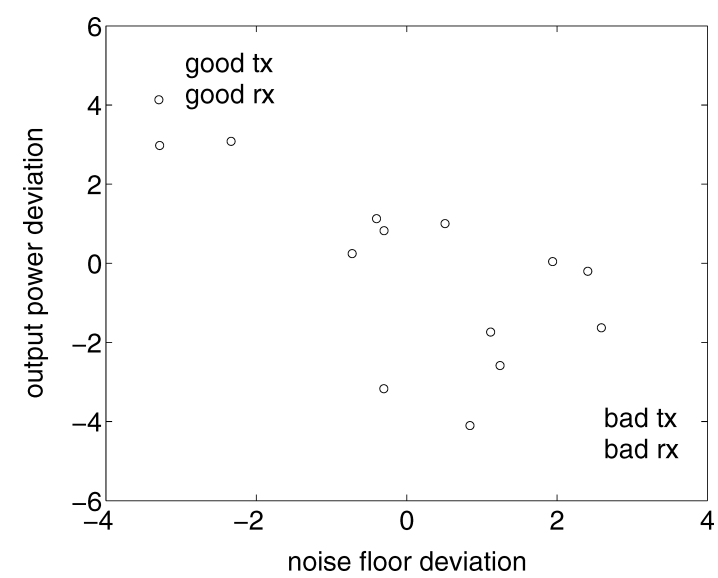

Fig. 10. Correlation between output power and noise floor.

and a high path-antenna gain will lead to higher output powers (i.e., good transmitter), and a lower noise floor (good receiver). Also, while our measurements where done in controlled scenarios, characteristics of real deployments, such as the remaining output power of batteries, may further enhance the correlation.

It is important to observe that this negative correlation leads to some nodes being good transmitters and receivers, which may create some cluster behavior, as observed in some empirical studies [Ganesan et al. 2003; Cerpa et al. 2005].

Negative correlation has a direct impact on the relation between the outdegree and in-degree of nodes. ${ }^{11}$ In Section 4.2 we had stated that the negative correlation between the output power and noise floor leads to the lowest level of link asymmetry, which implies that the in-degree and out-degree of nodes will be more similar than for positive and zero correlations. Figure 11 shows simulation results for the relation between in-degree and out-degree for positive and negative correlations between the output power and noise floor. The close relation between in-degree and out-degree for negative correlation can be observed. Figure 12 shows the empirical in-degree/out-degree relation of all nodes for all tested power levels, ${ }^{12}$ and we can observe that the empirical trend agrees with the simulation results. This close relation between in- and outdegree is highly desirable, given the strong dependance that several medium access and network-layer protocols have on symmetric links. Hence, from all

${ }^{11}$ In-degree is the number of neighbors that can communicate with a specific node, whereas outdegree is the number of neighbors that a specific node can communicate with.

${ }^{12}$ Links were considered valid if they had a PRR above $10 \%$. The same trend is observed for any blacklisting threshold. 
Table IV. Radio Parameters

\begin{tabular}{|ll|c|}
\hline & & (95\% conf. bounds) \\
\hline output power & $\sigma_{t x}$ & $2.3(1.7-3.5)$ \\
noise floor & $\sigma_{r x}$ & $1.9(1.4-3.1)$ \\
\hline
\end{tabular}

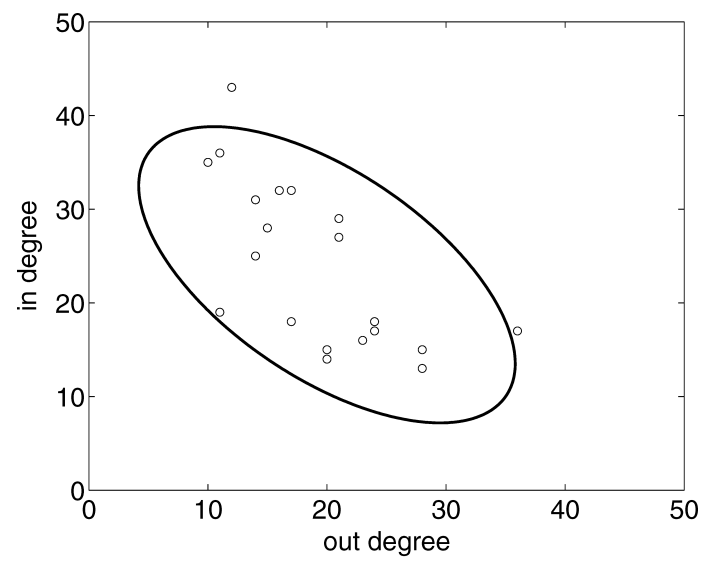

(a)

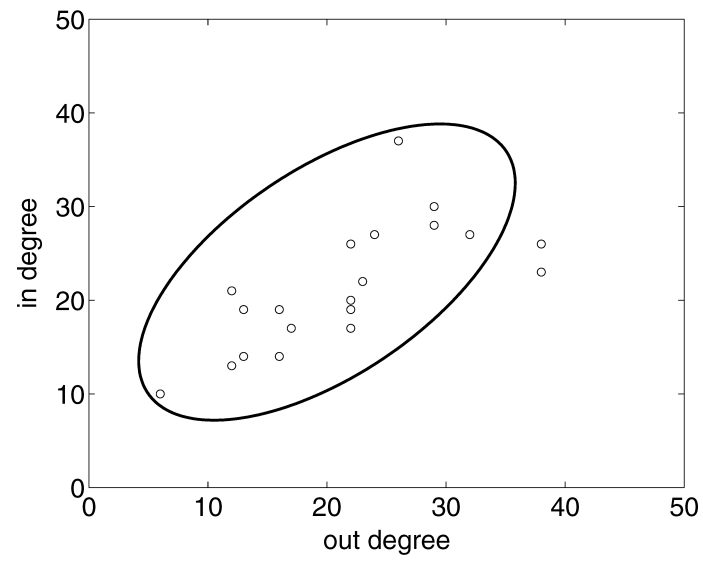

(b)

Fig. 11. Simulation results for the relation between in-degree and out-degree: (a) positive correlation; (b) negative correlation.

types of hardware variances a negative correlation between output power and noise floor is the least damaging in terms of link asymmetry.

-Noise floor: Given that our work does not consider interference, the noise floor can be obtained by the well-known thermal noise equation [Rappaport 2002], which leads to a value of $-115 \mathrm{dBm}$ for the parameters of the radio chip [Chipcon 2007]. However, our measurements showed that the average noise floor is approximately $-105 \mathrm{dBm}$; the $10 \mathrm{~dB}$ difference is mainly due to losses from the output pin of the chip to the antenna, which are not considered in the thermal noise equation. These losses depend on board implementation 


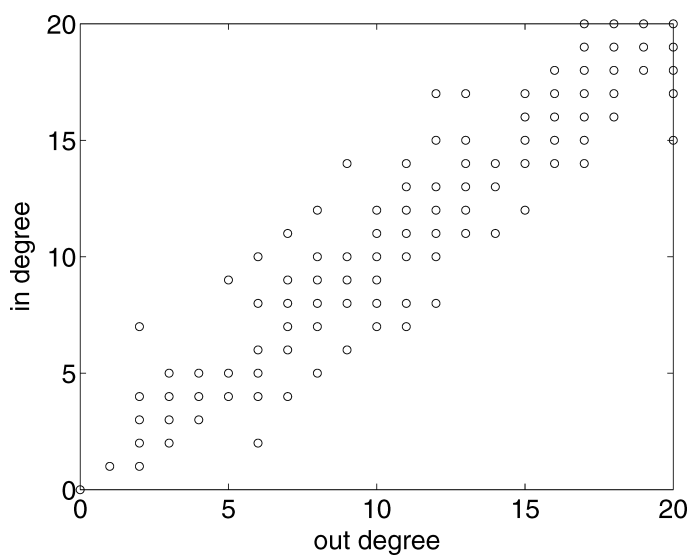

(a)

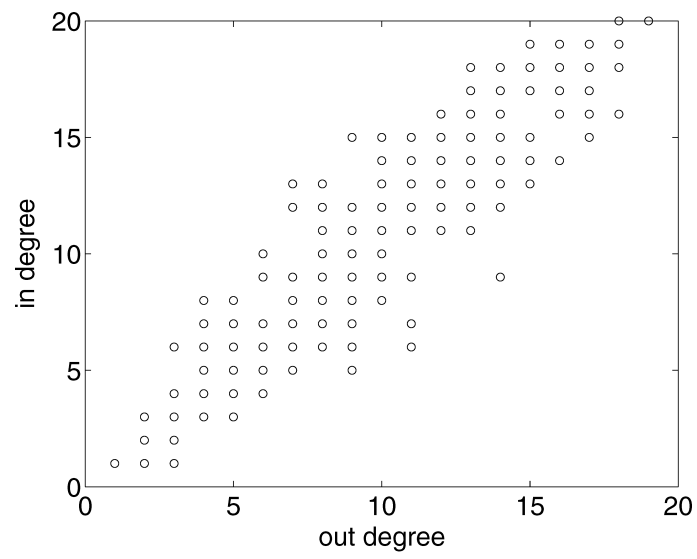

(b)

Fig. 12. Empirical correlation between in-degree and out-degree for different power levels: (a) indoor environment; (b) outdoor environment.

and are beyond the scope of this work. Hence, for the model the average noise floor $P_{n}$ will be set to $-105 \mathrm{dBm}$.

Finally, it is important to consider that bit-error rate that expressions are usually given in terms of $\frac{E_{b}}{N_{0}}$ (known as the SNR per bit). However, most commercially available radios provide only RSSI measurements which can be converted to SNR per packet $(\Upsilon)$. Note that $\Upsilon$ has a simple relation with $\frac{E_{b}}{N_{0}}: \Upsilon=\frac{E_{b}}{N_{0}} \frac{R}{B_{N}}$ for mica2 motes $R=19.2 \mathrm{kbps}$ (i.e., data rate) and $B_{N}=30 \mathrm{kHz}$ (noise bandwidth). Hence, all RSSI measurements can be converted to $\frac{E_{b}}{N_{0}}$ values.

\subsection{Chain Topologies}

For each environment, a chain topology of 21 motes was deployed with nodes spaced 1 meter apart. The frame size was 50 bytes with a preamble of 28 bytes. A simple TDMA protocol was implemented to avoid collisions. Every mote transmitted 100 packets at a rate of 5 packets/sec. Upon reception of a packet, the sequence number and received signal strength $\left(P_{r}\right)$ were stored; simultaneously, 
Table V. Comparison of $\sigma_{t}$

\begin{tabular}{|l|c|c|c|}
\hline & Expected $\sigma_{t}$ & Measured $\sigma_{t}$ & Measured $\sigma_{c h}$ \\
\hline indoor & 6.3 & 6.1 & 5.5 \\
outdoor & 4.8 & 5.1 & 3.2 \\
\hline
\end{tabular}

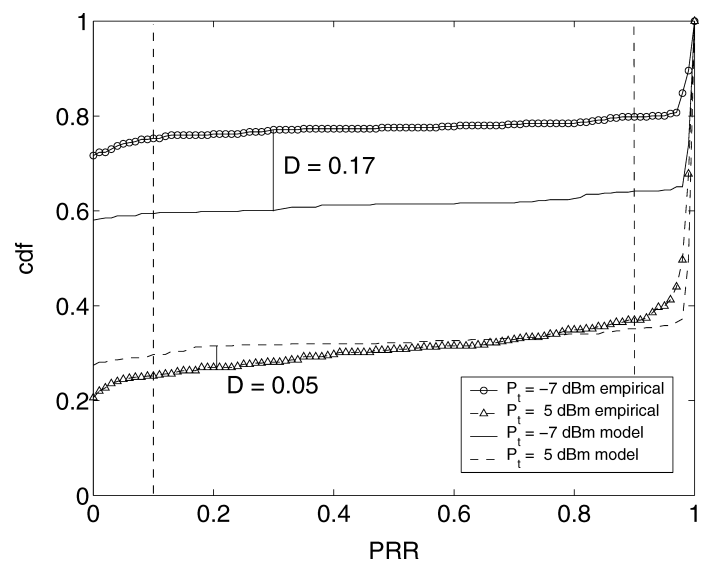

Fig. 13. Kolmogorov-Smirnov test between the empirical and model-generated packet reception rate for medium- and high-output powers in a grass area.

the noise floor was sampled. The average SNR and packet reception rate were measured for all links in the network.

According to Eq. (30), the expected total variance is the sum of channel and radio variances (sum of variances of Tables III and IV). Table V shows the expected $\sigma_{t}$, measured $\sigma_{t}$, and $\sigma_{c h}$. The expected $\sigma_{t}$ is derived from the $\sigma_{c h}$ and $\sigma_{h w}$ of Tables III and IV, and the measured $\sigma_{t}$ is obtained from the chain topology experiment. We observe that the expected and measured $\sigma_{t}$ are similar. It can also be observed that $\sigma_{c h}$ is smaller than $\sigma_{t}$ (especially for the outdoor environment), confirming that hardware variance contributes to the total variance, and consequently to the extent of the transitional region.

In order to validate our model, we present two comparisons. The first is a formal method based on the Kolmogorov-Smirnov (K-S) test and the second is a comparison of packet reception rate versus distance between empirical and simulated data. Figure 13 presents the cumulative distribution of the packet reception rate for the chain topology described earlier. Two power levels, namely $-7 \mathrm{dBm}$ and $5 \mathrm{dBm}$, are presented for the outdoor environment. For the number of links in the chain topology (i.e., 420) and a confidence interval of $10 \%$, the K-S table has a threshold value of 0.06 . For practical purposes let us define links with PRR above 0.9 as reliable and neglect links with PRR below 0.1. ${ }^{13}$ Then the distance $D$ of the K-S test is considered to be between 0.1 and 0.9 . We observe that low-density networks such as the medium-power case would not pass the test $(0.17>0.06)$, but high-density networks such as the highpower case do $(0.05<0.06)$, hence both distributions can be considered similar

${ }^{13} \mathrm{~A}$ very reasonable assumption, considering that links below 0.1 would incur significant losses or high numbers of retransmissions.

ACM Transactions on Sensor Networks, Vol. 3, No. 2, Article 7, Publication date: June 2007. 
(i.e., empirical and simulated). It is also important to notice that the empirical data shows that most of the probability mass is either above 0.9 or below 0.1 for any output power, as shown in Section 3.4. The next comparisons will illustrate why low-density networks are predicted less accurately than high-density ones.

Figure 14 shows the empirical packet reception rate versus distance compared to their analytical counterparts. It can be observed that the model provides a reasonable approximation of real behavior. Table VI shows the expected beginning and end of the transitional region, according to Eq. (8), for the scenarios in Figure 14. We can observe that this equation provides reasonable predictions for the empirical observations. Several power levels were tested, from $-20 \mathrm{dBm}$ to $5 \mathrm{dBm}$ in increments of $1 \mathrm{dBm}$, and all power levels showed similar behavior to the proposed model. A comparison of Figures 14(b) and (e) provides a better understanding of why the K-S test fails to asses both distributions as similar. In the simulated case (Figure 14(e)), the model tends to classify more links as good than does the empirical data. For instance, the simulated data shows that all links under a distance of 2 meters are considered to be 1.0, while the empirical data shows some unreliable links at a distance of 2 meters. Also, for distances 5 and 6 , some links are good $(>0.9)$ in the simulated data, while in the empirical data most are below 0.9. Since the transitional region is narrow, the disagreement on a few links leads to approximately $15 \%$ of the mass probability shifting from bad to good links, which causes the failure of the K-S test. Nevertheless, it is also worth considering that the major disagreement is only on differences at the extremes (good and bad links), while the slope for unreliable links is similar, showing that both empirical and modeled data have a close approximation on the number of unreliable links. It is important to mention that our model is not meant to be an exact replica of the environment, but an approximation.

\section{CONCLUSION}

The impact that channel and radio dynamics have on the performance of upperlayer protocols in wireless sensor networks requires a clear understanding of the behavior of the wireless link. The analysis presented allowed us to quantify the impact of channel multipaths and hardware variance on unreliable and asymmetric links. The main contributions of this work are:

- a systematic approach to obtain mathematical link-layer models for the statistical variation of packet reception rates with respect to distance. Model generators can be downloaded from http://ceng.usc.edu/ anrg/downloads. html; and

- analytical expressions for the boundaries of the transitional region were derived.

The advantage of our model is that the communication theory literature has channel parameters for different environments, and even though every environment is unique, these parameters can be used to obtain a first approximation of 


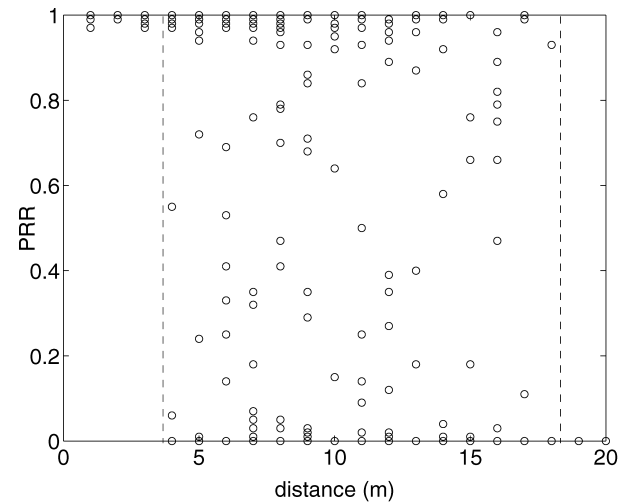

(a)

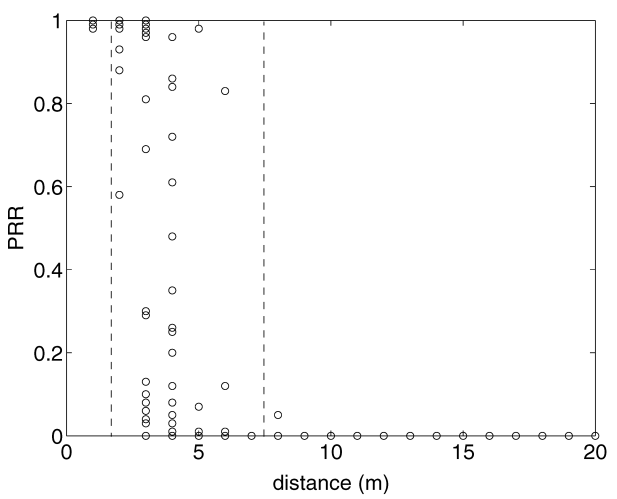

(b)

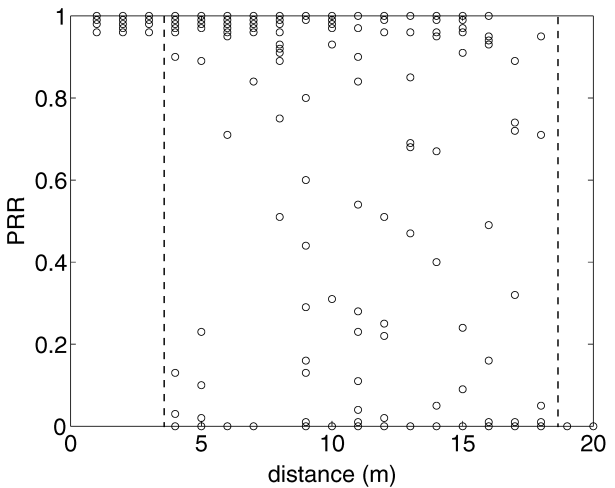

(c)

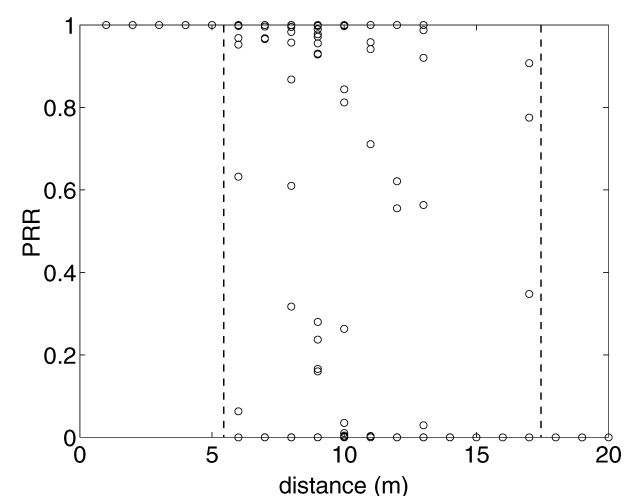

(d)

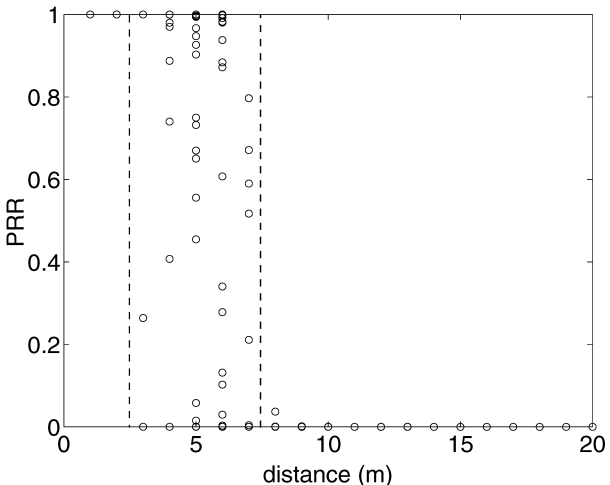

(e)

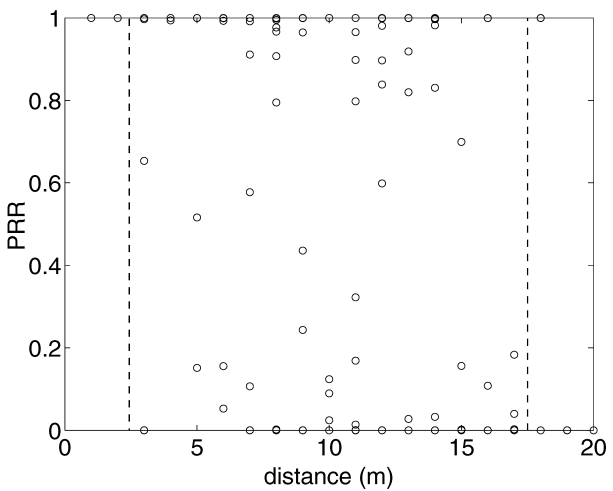

(f)

Fig. 14. Comparison of empirical measurements and instances of analytical model: (a) empirical indoor $P_{t}=-7 \mathrm{~dB}$; (b) empirical outdoor $P_{t}=-7 \mathrm{~dB}$; (c) empirical outdoor $P_{t}=5 \mathrm{~dB}$; (d), (e), and (f) are the analytical counterparts. 
Table VI. Analytical Extent of Transitional Region

\begin{tabular}{|l|c|c|}
\hline & Beginning $(\mathrm{m})$ & End $(\mathrm{m})$ \\
\hline indoor $P_{t}=-7 \mathrm{~dB}$ & 4.8 & 23.4 \\
outdoor $P_{t}=-7 \mathrm{~dB}$ & 3.4 & 8.1 \\
outdoor $P_{t}=5 \mathrm{~dB}$ & 6.1 & 14.6 \\
\hline
\end{tabular}

link behavior. If channel measurements are already available for the type of environment where the motes will be deployed, then there is no need to deploy the motes in situ to obtain the behavior of the link. The characteristics of the radio can be determined independently, that is, both receiver response (PRR versus SNR) and hardware variance, and when combined with the channel measurements, will give an approximation of the link behavior. In cases where there are no prior measurements of the channel, all the previous proposed models (including ours) require in situ measurements.

The key conclusions of our study are:

- The relative size of the transitional region ( $\Gamma$ coefficient) is higher for lower path loss exponents and higher variances;

-hardware variance induces a pseudovariance which increases the size of the transitional region;

-negative correlation between the output power and noise floor leads to lower levels of asymmetry, which is highly desirable, given the number of protocols that heavily depend on symmetric links;

-negative correlation also leads to nodes that are good transmitters and receivers, which helps explain the clustering behavior observed in previous works [Ganesan et al. 2003; Cerpa et al. 2005]; and

- even with a perfect-threshold radio, the transitional region still exists, so long as there are multipath effects.

Even though the simulations and empirical validation were based on radios using NC-FSK modulation and Manchester encoding, the model can be easily extended to other radio characteristics. Table VII presents the steps required for other common modulation techniques and encoding schemes.

It is important to highlight that while different modulations, encodings, and packet sizes lead to different sizes of regions, they do not significantly affect the $\Gamma$ coefficient, hence the results were not presented in the interest of space. Some of the results are presented in Zuniga and Krishnamachari [2004].

Our work contributes to a better understanding of the behavior of low-power wireless links, but is not exhaustive. It can be complemented with other studies to capture other important phenomena present in real scenarios; for instance, contention models from Son et al. [2006], temporal properties from Cerpa et al. [2005], and correlations due to direction of propagation from Zhou et al. [2006] (see Appendix A).

It is important to recall that physical-layer dynamics, appearing as the transitional region at the link layer, are not only potential causes of negative impact, 
Table VII. Theoretical Models for the Link Layer

\begin{tabular}{|c|c|}
\hline STEP 0: Radio & $\begin{array}{l}\text { Obtain output power and noise floor for all nodes } \\
\text { Can use Cholesky decomposition to generate } \\
\text { multivariate r.v. Eq. }(24) \\
\text { For mica2: }-20 \mathrm{dBm}<P_{t}<5 \mathrm{dBm}, P_{n}=-105 \mathrm{dBm}\end{array}$ \\
\hline STEP 1: Channel & $\begin{array}{l}\text { Obtain channel parameters } P L\left(d_{0}\right), n, \sigma \\
\text { Can be obtained through own empirical measurements, } \\
\text { or from some published results [Sohrabi et al. 1999] }\end{array}$ \\
\hline STEP 2: SNR & $\begin{array}{l}\text { Obtain SNR in } \mathrm{dB}\left(\gamma_{d B}\right) \text { as a function of distance } d \\
\gamma_{d B}(d)=T-P L\left(d_{0}\right)-10 \log _{10}\left(\frac{d}{d_{0}}\right)-N(0, \sigma)-R\end{array}$ \\
\hline STEP 3: Modulation & $\begin{array}{l}\text { Select modulation and insert } \gamma(d) \text { from previous step, } \\
\left.\text { but not in } \mathrm{dB} \text { (i.e., } \gamma=10^{\frac{\gamma d B}{10}}\right) \\
\text { Convert from } \frac{E_{b}}{N_{0}} \text { to } R S S I \text { by inserting appropriate } \\
\quad \text { bit data rate } R \text { and noise bandwidth } B_{N} \\
\text { According to modulation select appropriate BER }\left(P_{e}\right) \\
\text { ASK noncoherent: } \frac{1}{2}\left[\exp ^{-\frac{\gamma(d)}{2}} \frac{B_{N}}{R}+Q\left(\sqrt{\gamma(d) \frac{B_{N}}{R}}\right)\right] \\
\text { ASK coherent: } Q\left(\sqrt{\frac{\gamma(d)}{2} \frac{B_{N}}{R}}\right) \\
\text { FSK noncoherent: } \frac{1}{2} \exp ^{-\frac{\gamma(d)}{2}} \frac{B_{N}}{R} \\
\text { FSK coherent: } Q\left(\sqrt{\gamma(d) \frac{B_{N}}{R}}\right) \\
\text { PSK binary: } Q\left(\sqrt{2 \gamma(d) \frac{B_{N}}{R}}\right) \\
\text { PSK differential: } \frac{1}{2} \exp ^{-\gamma(d) \frac{B_{N}}{R}}\end{array}$ \\
\hline STEP 4: Encoding & $\begin{array}{l}\text { Select packet reception rate } \\
\text { Select according to encoding scheme, then insert frame, } \\
\text { preamble length, and } P_{e} \text { obtained in previous step } \\
\text { NRZ: }\left(1-P_{e}\right)^{8 \ell}\left(1-P_{e}\right)^{8(f-\ell)} \\
\text { 4B5B: }\left(1-P_{e}\right)^{8 \ell}\left(1-P_{e}\right)^{8(f-\ell) 1.25} \\
\text { Manchester: }\left(1-P_{e}\right)^{8 \ell}\left(1-P_{e}\right)^{8(f-\ell) 2.0} \\
\text { SECDED: }\left(1-P_{e}\right)^{8 \ell}\left(\left(1-P_{e}\right)^{8}+8 P_{e}\left(1-P_{e}\right)^{7}\right)^{(f-\ell) 3.0}\end{array}$ \\
\hline
\end{tabular}

but can have positive impact as well. For example, Miorandi and Altman [2005] and Bettstetter and Hartmann [2005] report that multipath effects have a positive impact on the connectivity of random topologies, and in Seada et al. [2004], an early version of the model presented in this article [Zuniga and Krishnamachari 2004] was used to obtain optimal forwarding distances for geographic routing. Hence, given the requirements of the application, our work provides a tool for the network designer to improve the overall performance of the protocols.

Finally, from preliminary results (see Appendix B), we find that even spread spectrum radios show transitional region effects; we therefore believe there is value in extending this work to other settings.

\section{APPENDIX A}

In Zhou et al. [2006] the authors present the degree of irregularity (DOI) coefficient as a mean to capture the variation per unit degree change in the direction 
of radio propagation. In that work the received power is given by

$$
\begin{aligned}
P_{r}(d) & =P_{t}-\text { DOIAdjustedPathLoss }+\mathcal{N}(0, \sigma) \\
& =P_{t}-\text { Path Loss } \times K_{i}+\mathcal{N}(0, \sigma),
\end{aligned}
$$

where $K_{i}$ is a coefficient to represent the difference in path loss in different directions, and the method to obtain it is presented in the RIM model [Zhou et al. 2006]. Hence, denoting $\overline{P L\left(d_{0}\right)}=P L\left(d_{0}\right)+10 \eta \log _{10}\left(\frac{d}{d_{0}}\right)$, Eq. (1) can be modified to include nonisotropic effects.

$$
P_{r}(d)=P_{t}-\overline{P L\left(d_{0}\right)} \times K_{i}+\mathcal{N}(0, \sigma)
$$

The effect of obstacles can be included by inserting a new variable on the previous equation. Let us denote denote $\Omega_{v w}$ as the path loss in $\mathrm{dB}$ due to an obstacle between nodes $v$ and $w$, for example, a wall. Then, letting $v$ be the transmitter, the received power at $w$ is given by

$$
P_{r_{w}}(d)=P_{t_{v}}-\left(\overline{P L\left(d_{0}\right)}+\Omega_{v w}\right) \times K_{i}+\mathcal{N}(0, \sigma) .
$$

Hence, if the layout of the environment is provided, the previous equation can be used to include additional path loss for each pair of nodes according to the obstacles between them.

\section{APPENDIX B}

Some preliminary empirical evaluations were done with micaZ devices. These motes have a $2.4 \mathrm{GHz}$ IEEE 802.15.4/ZigBee(tm) RF transceiver, which uses DSSS modem with $2 \mathrm{Mchips} / \mathrm{s}$ and $250 \mathrm{kbps}$ effective data rate. A chain topology with the same methodology as Section 5.2 was deployed in the same indoor environment as mica2 motes.

Figure 15 presents empirical measurements for the channel, radio, and link for mica2 and micaZ. The nominal output power for both types of motes was $-10 \mathrm{dBm}$. We observe that the transitional region still has a significant extent. However, for the same output power, micaZ radios seem to have larger connected and transitional regions.

No major differences were found in the shadowing standard deviation for both deployments (i.e., around 6.1 for both). However, the path loss exponent for micaZ measurements is $1.94(1.65,2.23)$, which is smaller than the corresponding value for mica2 in Table III $(\eta=3.3)$. According to Eq. (8), a smaller $\eta$ increases the size of both regions, which provides some intuition as to why the extent of the regions is larger for micaZ motes for the same output power.

Spread spectrum techniques seem to partially combat multipath by decreasing $\eta$, consequently providing larger coverage for the same output power. However, as stated in Eq. (9), a lower $\eta$ implies a larger transitional region, which increases the number of unreliable and asymmetric links. An in-depth study of the impact of low-cost spread spectrum radios in the transitional region is part of our future work. 


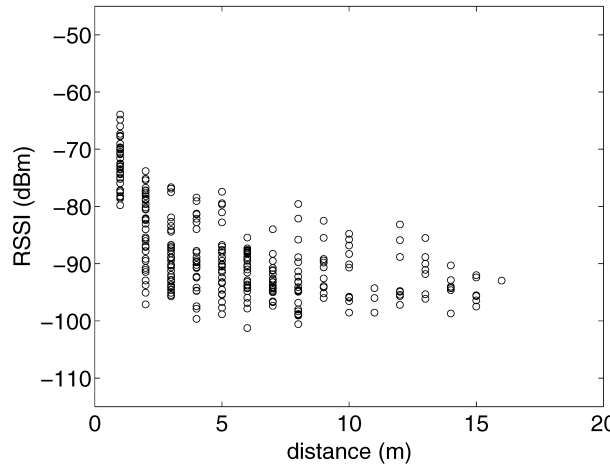

(a)

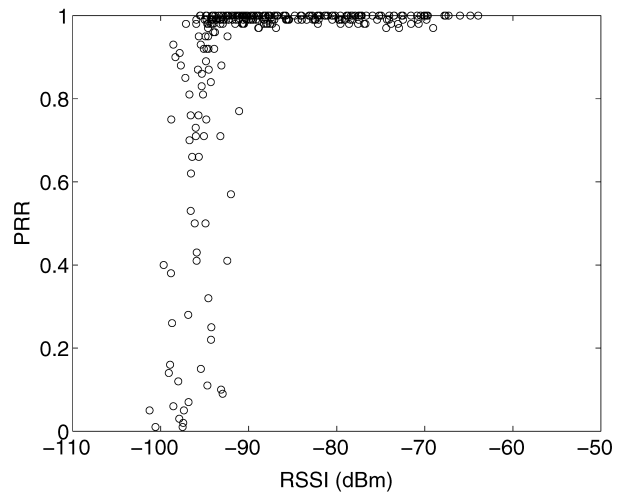

(b)

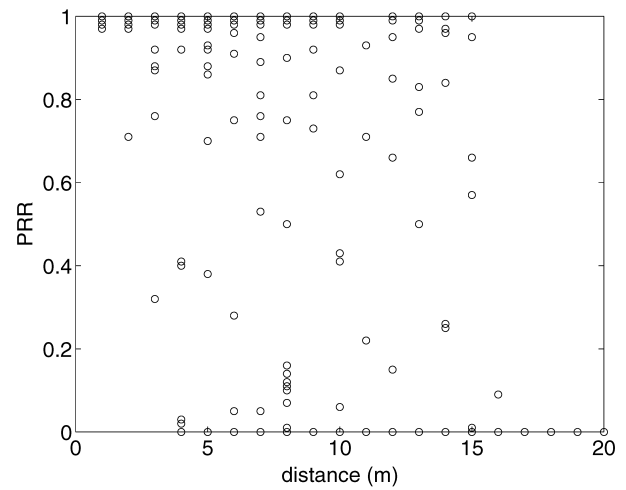

(c)

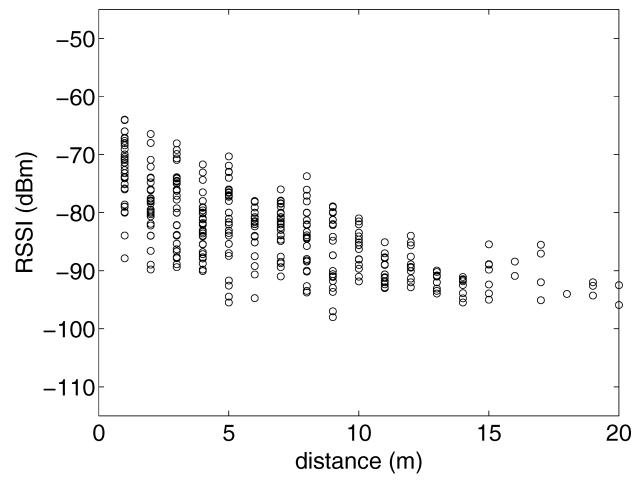

(d)

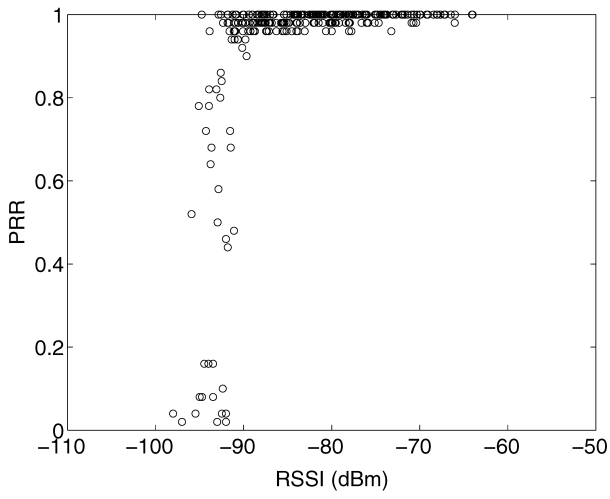

(e)

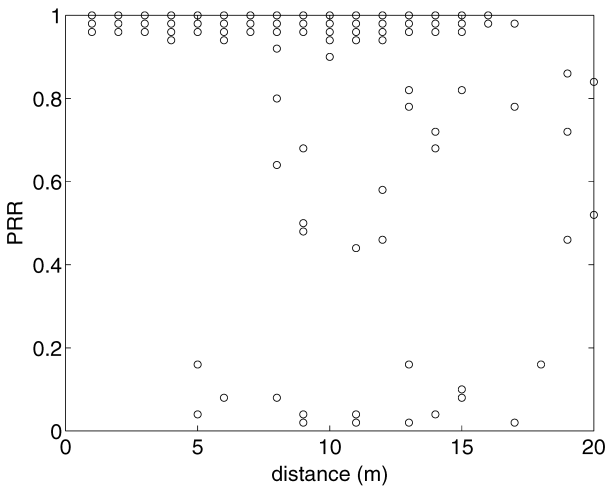

(f)

Fig. 15. Comparison of empirical measurements for channel, radio, and link between mica2 and micaZ motes, $P_{t}=-10 \mathrm{dBm}$ for both type of motes: (a) channel mica2; (b) radio mica2; (c) link mica2; (d), (e), and (f) are their micaZ counterparts. 


\section{ACKNOWLEDGMENTS}

The authors would like to acknowledge assistance from and useful conversations with Urbashi Mitra, Alec Woo, Jerry Zhao, John Heidemann, Robert Poor, and Scott Shenker.

\section{REFERENCES}

Bettstetter, C. and Hartmann, C. 2005. Connectivity of wireless multihop networks in a shadow fading environment. Wireless Netw. 11, 5, 571-579.

Cerpa, A., Busek, N., ANd Estrin, D. 2003. SCALE: A tool for simple connectivity assessment in lossy environments. Tech. Rep. CENS, University of California, Los Angeles.

Cerpa, A., Wong, J., Kuang, L., Potkonjak, M., and Estrin, D. 2005. Statistical model of lossy links in wireless sensor networks. In Proceedings of the 4th International Symposium on Information Processing in Sensor Networks.

Cerpa, A., Wong, J., Potkonjak, M., And Estrin, D. 2005. Temporal properties of low power wireless links: Modeling and implications on multi-hop routing. In Proceedings of the 6th ACM International Symposium on Mobile Ad Hoc Networking and Computing. 414-425.

Chipcon. 2007. cc1000 low power radio transceiver. www.chipcon.com/index.cfm?kat_id=2\& subkat_id=12\&dokid=14.

De Couto, D., Aguayo, D., Bicket, J., And MorRis, R. 2005. A high-throughput path metric for multi-hop wireless routing. Wireless Netw. 11, 4, 419-434.

Ganesan, D., Krishnamachari, B., Woo, A., Culler, D., Estrin, D., and Wicker, S. 2003. Complex behavior at scale: An experimental study of low-power wireless sensor networks. Tech. Rep. UCLA/CSD-TR, 02-0013, Department of Computer Science, University of California at Los Angeles.

Girod, L., Elson, J., Cerpa, A., Stathopoulos, T., Ramanathan, N., and Estrin, D. 2004. Emstar: A software environment for developing and deploying wireless sensor networks. In Proceedings of the USENIX Technical Conference.

Kotz, D., Newport, C., AND ElLiott, C. 2003. The mistaken axioms of wireless-network research. Tech. Rep. Department of Computer Science, Dartmouth College. July.

Levis, P., Lee, N., Welsh, M., AND Culler, D. 2003. TOSSIM: Accurate and scalable simulation of entire tinyOS applications. In Proceedings of the 1st International Conference on Embedded Networked Sensor Systems. 126-137.

Mionandi, D. AND Altman, E. 2005. Coverage and connectivity of ad hoc networks in presence of channel randomness. In Proceedings of the 24th Annual Joint Conference of the IEEE Computer and Communications Societies.

NikoOKAR, H. AND HASHEMI, H. 1993. Statistical modeling of signal amplitude fading of indoor radiopropagation channels. Universal Personal Communications. 'Personal Communications: Gateway to the 21st Century'. Conference Record., 2nd International Conference.

Poor, R. 2004. Sources of asymmetry in wireless mesh sensor networks. Private communication.

RAPPAPORT, T. 2002. Wireless Communications. Prentice-Hall, Upper Saddle River, NJ.

Seada, K., Zuniga, M., Helmy, A., and Krishnamachari, B. 2004. Energy-Efficient forwarding strategies for geographic routing in lossy wireless sensor networks. In Proceedings of the 2nd International Conference on Embedded Networked Sensor Systems. 108-121.

SEIDEL, S. Y. AND RAPPAPORT, T. S. $1992.914 \mathrm{MHz}$ path loss prediction model for indoor wireless communication in multi floored buildings. IEEE Trans. Antennas Propagation 40, 2, 207-217.

Sohrabi, K., Manriquez, B., and Pottie, G. 1999. Near ground wideband channel measurement in 800-1000 MHz. In Proceedings of the IEEE 49th Vehicular Technology Conference.

Son, D., Krishnamachari, B., AND Heidemann, J. 2004. Experimental study of the effects of transmission power control and blacklisting in wireless sensor networks. In Proceedings of the IEEE 1st Annual Conference on Sensor and Ad Hoc Communications and Networks (SECON). 289-298.

Son, D., Krishnamachari, B., ANd Heidemann, J. 2006. Experimental analysis of concurrent packet transmissions in low-power wireless networks. In Proceedings of the 4th International Conference on Embedded Networked Sensor Systems. 
Woo, A., Tong, T., And Culler, D. 2003. Taming the underlying challenges of reliable multihop routing in sensor networks. In Proceedings of the 1st International Conference on Embedded Networked Sensor Systems. 14-27.

ZhaO, J. AND Govindan, R. 2003. Understanding packet delivery performance in dense wireless sensor networks. In Proceedings of the 1st International Conference on Embedded Networked Sensor Systems. 1-13.

Zhou, G., He, T., Krishnamurthy, S., AND Stankovic, J. 2006. Models and solutions for radio irregularity in wireless sensor networks. ACM Tran. Sensor Netw. 2, 2, 221-262.

Zuniga, M. AND KRISHNAMACHARI, B. 2004. Analyzing the transitional region in low power wireless links. In Proceedings of the IEEE 1st Annual Conference on Sensor and Ad Hoc Communications and Networks (SECON). 517-526.

Received April 2006; revised October 2006; accepted January 2007 